\title{
FIR Filters for Online Trajectory Planning with Time- and Frequency-Domain Specifications
}

\author{
Luigi Biagiotti ${ }^{\mathrm{a}, *}$, Claudio Melchiorri ${ }^{\mathrm{b}}$ \\ ${ }^{a}$ Department of Information Engineering (DII), University of Modena and Reggio \\ Emilia, Via Vignolese 905, 41100 Modena, Italy \\ ${ }^{b}$ Department of Electronics, Informatics and Systems (DEIS), University of \\ Bologna, Viale Risorgimento 2, 40136 Bologna, Italy
}

\begin{abstract}
In this paper, the use of FIR (Finite Impulse Response) filters for planning minimum-time trajectories for robots or automatic machines under constraints of velocity, acceleration, etc. is presented and discussed. In particular, the relationship between multi-segment polynomial trajectories, i.e. trajectories composed of several polynomial segments, each one possibly characterized by constraints on one or more specific derivatives (i.e. velocity, acceleration, jerk, etc.), and FIR filters disposed in a cascade configuration is demonstrated and exploited in order to design a digital filter for online trajectory planning. The connection between analytic functions and dynamic filters allows a generalization of these trajectories, usually obtained by second- or third-order polynomial functions (e.g. trapezoidal velocity and double S velocity trajectories), to a generic order with only a modest increase of the complexity. As a matter of fact, the computation of trajectories
\end{abstract}

\footnotetext{
${ }^{*}$ Corresponding author. Tel.:+390592056315; fax:+390592056329.

Email addresses: luigi.biagiotti@unimore.it (Luigi Biagiotti), claudio.melchiorri@unibo.it (Claudio Melchiorri)
} 
with higher degree of continuity simply requires additional FIR filters in the chain. Moreover, the modular structure of the planner provides a direct frequency characterization of the motion law. In this way, it is possible to define the trajectories by considering constraints expressed in the frequency-domain besides the classical time-domain specifications, such as bounds on velocity, acceleration, and so on. Two examples illustrate the main features of the proposed trajectory planner, in particular with respect to the problems of multi-point trajectories generation and residual vibrations suppression.

Keywords: Trajectory planning, Multi-segment trajectories, Shaping filters, Digital filters

\section{Introduction}

The growing need of planning trajectories online has led to the development of a number of filters able to produce motion profiles with the desired degree of smoothness simply starting from rough reference signals, such as step functions, which set the desired final position. Examples of these trajectory planners have been presented e.g. in Zanasi et al. (2000); Zanasi and Morselli (2003) or, more recently, in Zheng et al. (2009), where minimumtime trajectory planners with bounds on velocity, acceleration, and jerk have been proposed. Basically, these planners are composed by a chain of integrators (whose output represents the desired trajectory) with a proper nonlinear feedback controller so that the reference input is tracked in the fastest possible manner while remaining compliant with the given constraints. These trajectory generators allow to consider even asymmetric positive and negative limits, that can be changed in runtime. Moreover, the filters can be applied 
to reference signals different from simple step functions. In this case, the output follows the input with no changes and no delay if the input is compliant with the constraints, otherwise the output tracks the input at best under the imposed limits. This kind of filters has been successfully applied in the robotics field, in order to cope with the speed limits that characterize mobile robots, see Bonfè and Secchi (2010), and to take into account the torque limits affecting mechanical manipulators, see Gerelli and Guarino Lo Bianco (2008), besides the usual kinematic bounds on velocity and acceleration, as in Gerelli and Guarino Lo Bianco (2009). However, although very versatile, these trajectory planners are characterized by an high complexity and therefore are rather demanding from a computational point of view. A simpler solution to the problem of online trajectory planning and trajectory smoothing consists in the application to the reference commands, provided by a coarse interpolator, of one or more linear filters. In motion control of CNC machines, FIR (Finite Impulse Response) filters are generally adopted because their efficiency and the possibility to be easily implemented by hardware. The acceleration/deceleration circuit proposed in Nozawa et al. (1985) is nothing but a moving average filter that produces as output the mean of the last $n$ input samples (of the reference velocity). In Kim et al. (1994) the convolution of the reference signal, representing the velocity along the desired path, with various kinds of digital filters is proposed for properly shaping the acceleration/deceleration profile; again, a single moving average filter is used to obtain a constant acceleration but it is recognized that a chain of such filters would make the motion smoother and smoother. In Jeon and $\mathrm{Ha}$ (2000) the use of a single FIR filter for smoothing a given feedrate 
signal is generalized to any kind of acceleration profile, by properly computing coefficients of the filter. A similar approach, but based on continuous filters, is represented by the so-called input shaping, that consists in filtering the reference input by convolving it with a train of impulses in order to form a new command that causes little or no vibrations on the mechanical plant, see Singer and Seering (1990); Tuttle and Seering (1994). This technique has been adopted for reduction of crane oscillations, see Hong and Hong (2004), control of industrial machines like XY stages, see Fortgang et al. (2005), vibration suppression in flexible robotic arms, see Magee and Book (1998). For a comprehensive overview about input shaping techniques refer to Singhose (2009).

In this paper, the advantages of the filtering techniques, that allow to properly shape the frequency spectrum of a motion law, are combined with the features of multi-segment trajectories, whose parameters are generally defined with the only purpose of making the trajectories compliant with given bounds on velocity, acceleration, jerk, etc. The key point is the equivalence between time-optimal multi-segment polynomial trajectories with constraints on the first $n$ derivatives and the output of a chain of $n$ moving average filters. Therefore, in this case the filters are not used for making a given trajectory smoother but for online generating a trajectory starting from initial and final positions, similarly to feedback controlled planners. The equivalence between dynamic filters and trajectories expressed by analytic functions provides an immediate characterization of the motion from a spectral point of view. This is of great importance when it is necessary to plan a trajectory for systems which are critical with respect to the problem of vibrations (Lambrechts 
et al., 2005; Barre et al., 2005), since it is possible to set the parameters of the trajectory on the basis of the frequency response of the plant.

The paper is organized as follows. In Sec. 2 the equivalence between multisegment trajectories defined by analytic functions and the output of chains of finite memory filters is demonstrated. On the basis of this equivalence, the formulae relating the characteristic parameters of the filters and the limit values of velocity, acceleration, jerk, etc. are deduced and the spectrum of a generic trajectory of order $n$ is obtained. In Sec. 3 the continuous-time filters are approximated by discretization with banks of moving average filters that can be directly implemented on digital controllers. Section 4 illustrates, by means of some numerical examples, the advantages of the proposed filter for generating multi-point trajectories and planning time-optimal motion profiles in those applications in which, besides bounds on the magnitude of trajectory derivatives, constraints in the frequency domain are present. Concluding remarks are provided in the last section.

\section{Multi-segment trajectories and dynamic filters}

Multi-segment trajectories are motion laws composed by several tracts, each one characterized by a specific analytical expression, properly joined in order to guarantee the desired degree of smoothness. In particular, timeoptimal trajectories under constraints of velocity, acceleration, jerk, etc. are characterized by segments in which the velocity, the acceleration, and higher derivatives (depending on the required order of continuity) are saturated to the maximum allowed value. By imposing constraints on the first $n$ derivatives one obtains a trajectory $q(t)$ of $\operatorname{class} \mathcal{C}^{n-1}$, that is with the first $n-1$ 


$$
\stackrel{h u(t)}{\longrightarrow} \frac{1-e^{-s T_{1}}}{s T_{1}} \stackrel{q_{1}(t)}{\longrightarrow} \frac{1-e^{-s T_{2}}}{s T_{2}} \stackrel{q_{2}(t)}{\longrightarrow} \stackrel{q_{n-1}(t)}{\longrightarrow} \frac{1-e^{-s T_{n}}}{s T_{n}} \stackrel{q_{n}(t)}{\longrightarrow}
$$

Figure 1: System composed by $n$ filters for the computation of an optimal trajectory of $\operatorname{class} \mathcal{C}^{n-1}$.

derivatives that are continuous, while the $n$-th derivative $q^{(n)}(t)$ is a piecewise constant function whose values belong to the set $\left\{q_{\min }^{(n)}, 0, q_{\max }^{(n)}\right\}$. The number $n$ is called order of the trajectory. Typical examples of multi-segment trajectories are the well known "trapezoidal velocity" trajectory and the "double S velocity" trajectory, of order two and three respectively. With the additional condition of symmetric constraints:

$$
q_{\min }^{(i)}=-q_{\max }^{(i)}, \quad i=1, \ldots, n
$$

one can show that such a kind of trajectories can be obtained by filtering a step input with a cascade of $n$ dynamic filters, each one characterized by the transfer function

$$
M_{i}(s)=\frac{1}{T_{i}} \frac{1-e^{-s T_{i}}}{s}
$$

where the parameter $T_{i}$ (in general different for each filter composing the chain) is a time length, see Fig. 1. The possibility of obtaining time-optimal trajectories with the system of Fig. 1 fed by step input functions can be proved by exploiting a property of the convolution product (denoted with $*$ ) on the differentiation, i.e.

$$
\frac{d}{d t}(f * g)=\frac{d f}{d t} * g=f * \frac{d g}{d t}
$$

Consider the case of a single filter with a step input of generic magnitude 


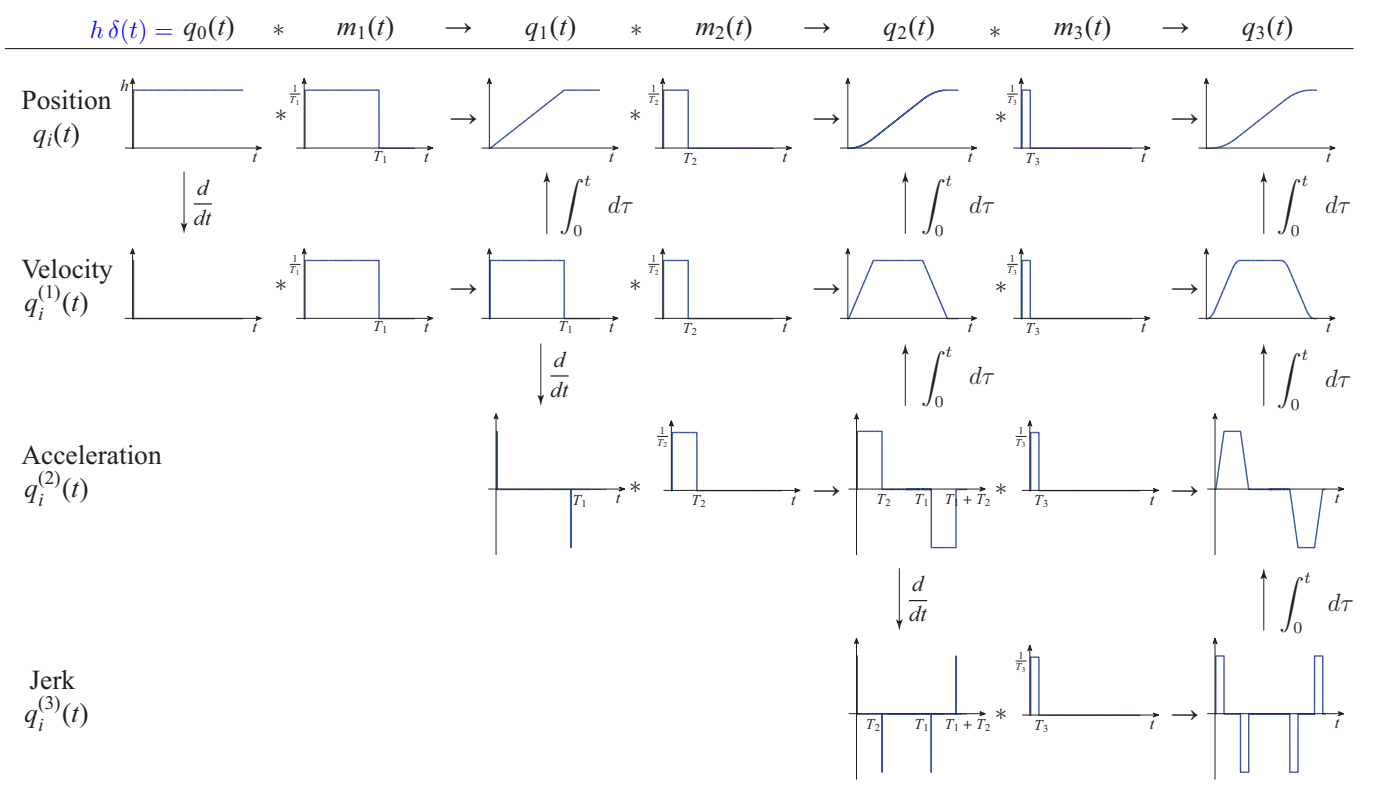

Figure 2: Relationships among the profiles of trajectories obtained by iterated averaging operations. Note that in the first row the algebraic relation $q_{i}(t)=q_{i-1}(t) * m_{i}(t), i=1,2,3$ is reported, while in the remaining rows a pictorial representation of the relationship among the trajectories of different orders and their derivatives is shown.

$h$, i.e. $h u(t)$, being $u(t)$ the unit step function

$$
u(t)= \begin{cases}1, & t \geq 0 \\ 0, & t<0 .\end{cases}
$$

In this case the output trajectory can be computed as

$$
q_{1}(t)=h u(t) * m_{1}(t)
$$

where

$$
m_{i}(t)=\mathcal{L}^{-1}\left\{M_{i}(s)\right\}=\frac{1}{T_{i}}\left(u(t)-u\left(t-T_{i}\right)\right), \quad i=1
$$

is the impulse response corresponding to $M_{i}(s)$. Note that $m_{i}(t)$ is a rectangular function of duration $T_{i}$ and magnitude $1 / T_{i}$, see Fig. 2. This implies 
that, as well known, for any choice of $T_{i}$ the area of the rectangular function is unitary, and accordingly the static gain of the corresponding function $M_{i}(s)$ is unitary as well:

$$
M_{i}(0)=\int_{0}^{\infty} m_{i}(\tau) d \tau=1
$$

By applying (2) to (3) one obtains

$$
\begin{aligned}
q_{1}^{(1)}(t) & =h u^{(1)}(t) * m_{1}(t) \\
& =h \delta(t) * m_{1}(t)=h m_{1}(t)
\end{aligned}
$$

where $\delta(t)$ is the unit impulse function. Therefore, by adopting a single filter $M_{1}(s)$ fed by a step function of amplitude $h$, the output consists in a trajectory $q_{1}(t)$ whose velocity has a rectangular profile with magnitude $\mathrm{v}=h / T_{1}$. Then, it is immediate to obtain the value of the parameter $T_{1}$ which permits to impose a desired (limit) value of the velocity ${ }^{1}$ :

$$
\mathrm{v}=\frac{|h|}{T_{1}}=q_{\max }^{(1)} \rightarrow T_{1}=\frac{|h|}{q_{\max }^{(1)}} .
$$

Accordingly, when a step input of amplitude $h$ is applied, the output of $M_{1}(s)$ will change from the initial to the final value (given by $h$ ) with a linear profile whose duration is exactly $T_{1}$.

If one adds a second filter $M_{2}(s)$, characterized by the parameter $T_{2}$, the

\footnotetext{
${ }^{1}$ Since $T_{1}$ must be positive, it is necessary to consider the absolute value of the displacement. In fact, if $h<0$ the constant velocity will be equal to the minimum value, that is

$$
\mathrm{v}=\frac{h}{T_{1}}=q_{\min }^{(1)}=-q_{\max }^{(1)} \quad \rightarrow \quad T_{1}=\frac{-h}{q_{\max }^{(1)}}=\frac{|h|}{q_{\max }^{(1)}}
$$
}


resulting trajectory is

$$
\begin{aligned}
q_{2}(t) & =q_{1}(t) * m_{2}(t) \\
& =h u(t) * m_{1}(t) * m_{2}(t) .
\end{aligned}
$$

Therefore, the first derivative is

$$
\begin{aligned}
q_{2}^{(1)}(t) & =q_{1}^{(1)}(t) * m_{2}(t) \\
& =h m_{1}(t) * m_{2}(t)
\end{aligned}
$$

and, by taking into account that

$$
m_{1}^{(1)}(t)=\frac{1}{T_{1}}\left(\delta(t)-\delta\left(t-T_{1}\right)\right)
$$

it is possible to deduce the second derivative

$$
\begin{aligned}
q_{2}^{(2)}(t) & =h m_{1}^{(1)}(t) * m_{2}(t) \\
& =\frac{h}{T_{1}}\left(\delta(t)-\delta\left(t-T_{1}\right)\right) * m_{2}(t) \\
& =\mathrm{v}\left(m_{2}(t)-m_{2}\left(t-T_{1}\right)\right)
\end{aligned}
$$

which is composed by two rectangular functions, one positive and one negative, of magnitude $a=\frac{\mathrm{v}}{T_{2}}$ and duration $\min \left\{T_{1}, T_{2}\right\}$. Therefore the maximum value of the acceleration can be freely set by imposing

$$
\mathrm{a}=\frac{\mathrm{v}}{T_{2}}=q_{\max }^{(2)} \rightarrow T_{2}=\frac{\mathrm{v}}{q_{\max }^{(2)}}=\frac{q_{\max }^{(1)}}{q_{\max }^{(2)}} .
$$

Since the static gain of both $M_{1}(s)$ and $M_{2}(s)$ is unitary, the final value of the response of $M_{1}(s) \cdot M_{2}(s)$ to a step input of magnitude $h$ remains $h$. The system output $q_{2}(t)$ reaches such a value with a trapezoidal velocity profile obtained by integrating $q_{2}^{(2)}(t)$. 
The maximum acceleration of the trajectory is $q_{\max }^{(2)}$, and the velocity is still limited by $q_{\max }^{(1)}$. In fact, by defining for a generic function $f(t)$

$$
\operatorname{peak}(f(t))=\max _{t \geq 0}|f(t)|
$$

from (6) one can prove that

$$
\begin{aligned}
\operatorname{peak}\left(q_{2}^{(1)}(t)\right) & \leq \operatorname{peak}\left(q_{1}^{(1)}(t)\right) \cdot \int_{0}^{\infty}\left|m_{2}(\tau)\right| d \tau \\
& \leq \operatorname{peak}\left(q_{1}^{(1)}(t)\right)=q_{\text {max }}^{(1)}
\end{aligned}
$$

being $\int_{0}^{\infty}\left|m_{2}(\tau)\right| d \tau=\int_{0}^{\infty} m_{2}(\tau) d \tau=1$ since $m_{2}(t) \geq 0, \forall t$. In this case, if $T_{1} \geq T_{2}$ then the maximum velocity $q_{\max }^{(1)}$ is actually reached, i.e. $\operatorname{peak}\left(q_{2}^{(1)}(t)\right)=$ $q_{\text {max }}^{(1)}$ and $q_{2}(t)$ is a minimum-time trajectory compliant with the given bounds $q_{\max }^{(i)}, i=1,2$. Conversely, if $T_{1}<T_{2}$ then peak $\left(q_{2}^{(1)}(t)\right)=\frac{|h|}{T_{2}}<\frac{|h|}{T_{1}}=q_{\max }^{(1)}$, and the trajectory, that still meets the proposed constraints, is not of minimum duration. In Fig. 3, the trapezoidal trajectories obtained for two different displacements $h$ and with the same limits $q_{\max }^{(1)}$ and $q_{\max }^{(2)}$ that guarantee $T_{1}=0.08 \geq T_{2}=0.05$ in case (a) and $T_{1}=0.02<T_{2}=0.05$ in case (b) are reported. Note in particular, that when $T_{1}<T_{2}$, the roles of the two time constants $T_{i}$ are switched (in the sense that the duration of the acceleration period is $T_{1}$ and the maximum velocity is $h / T_{2}$ ).

The total duration of the trajectory $q_{2}(t)$ is given by the sum of the durations of the impulse responses of $M_{1}(s)$ and $M_{2}(s)$, i.e.

$$
T_{\text {tot }}=T_{1}+T_{2}
$$

Note that the maximum velocity $q_{\max }^{(1)}$ is actually reached if and only if

$$
T_{2} \leq \frac{1}{2} T_{t o t}=\frac{1}{2}\left(T_{1}+T_{2}\right) \quad \Leftrightarrow \quad T_{2} \leq T_{1}
$$




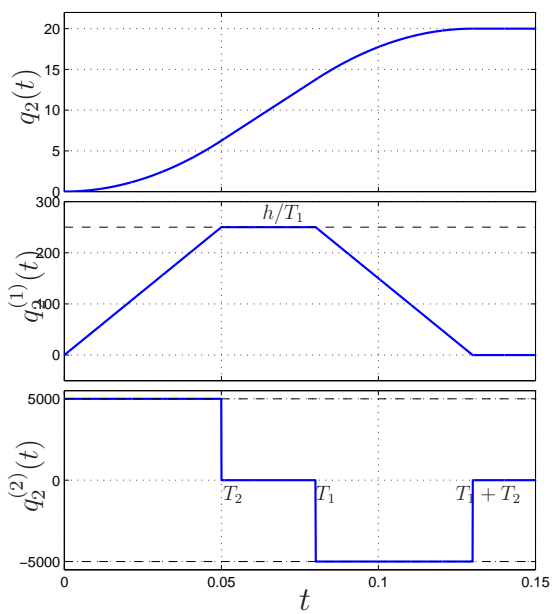

(a)

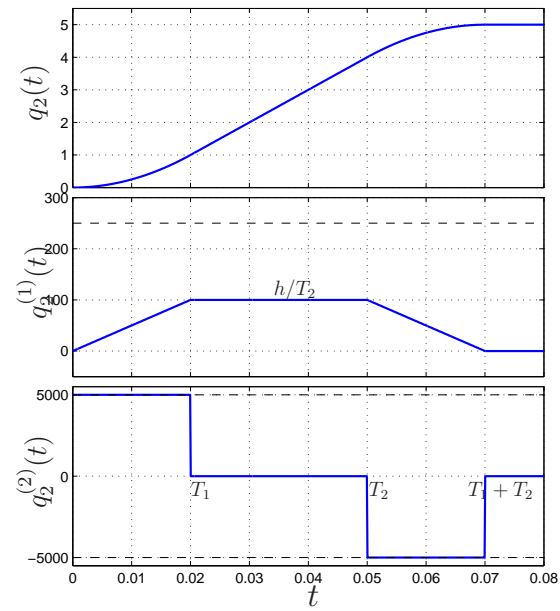

(b)

Figure 3: Trapezoidal velocity trajectories $q_{2}(t)$ obtained with the constraints $q_{\max }^{(1)}=250$ $\mathrm{rad} / \mathrm{s}$ and $q_{\max }^{(2)}=5000 \mathrm{rad} / \mathrm{s}^{2}$ and $h=20 \mathrm{rad}(\mathrm{a}), h=5 \mathrm{rad}(\mathrm{b})$.

that is if and only if the (planned) duration $T_{2}$ of the acceleration/deceleration period is not greater than half of the total duration of the trajectory. As shown in Fig. 2, the second order trajectory $q_{2}(t)$ can be made smoother by adding a further filter $M_{3}(s)$ (characterized by the parameter $T_{3}$ ), obtaining in this way a double $\mathrm{S}$ velocity trajectory

$$
q_{3}(t)=q_{2}(t) * m_{3}(t)
$$

whose velocity, acceleration and jerk are respectively

$$
\begin{aligned}
& q_{3}^{(1)}(t)=q_{2}^{(1)}(t) * m_{3}(t) \\
& q_{3}^{(2)}(t)=q_{2}^{(2)}(t) * m_{3}(t) \\
& q_{3}^{(3)}(t)=q_{2}^{(3)}(t) * m_{3}(t) .
\end{aligned}
$$

Since $q_{2}^{(2)}(t)$ is composed by two rectangular functions, its derivative is a sequence of four impulsive functions of amplitude a properly shifted in time, 
see Fig. 2. Therefore, from (9) it descends that $q_{3}^{(3)}(t)$ is composed by four rectangular functions of amplitude $\mathrm{j}=\mathrm{a} / T_{3}$ and accordingly it is possible to select $T_{3}$ on the basis of the desired value of the jerk:

$$
\mathrm{j}=\frac{\mathrm{a}}{T_{3}}=q_{\max }^{(3)} \rightarrow T_{3}=\frac{\mathrm{a}}{q_{\max }^{(3)}}=\frac{q_{\max }^{(2)}}{q_{\max }^{(3)}} .
$$

Moreover, by the same argument as in (8) one can prove that

$$
\begin{aligned}
& \operatorname{peak}\left(q_{3}^{(2)}(t)\right) \leq \operatorname{peak}\left(q_{2}^{(2)}(t)\right)=q_{\text {max }}^{(2)} \\
& \operatorname{peak}\left(q_{3}^{(1)}(t)\right) \leq \operatorname{peak}\left(q_{2}^{(1)}(t)\right) \leq \operatorname{peak}\left(q_{1}^{(1)}(t)\right)=q_{\text {max }}^{(1)}
\end{aligned}
$$

In particular, if the tract with constant jerk is at most half of the acceleration/deceleration period, that is

$$
T_{3} \leq \frac{1}{2}\left(T_{2}+T_{3}\right) \quad \Leftrightarrow \quad T_{3} \leq T_{2}
$$

in (11) the sign equal holds true and the maximum acceleration $q_{\max }^{(2)}$ is actually reached by the third order trajectory $q_{3}(t)$. Analogously, if the acceleration/deceleration period does not exceed half of the total duration of the trajectory, i.e.

$$
T_{2}+T_{3} \leq \frac{1}{2}\left(T_{1}+T_{2}+T_{3}\right) \quad \Leftrightarrow \quad T_{2}+T_{3} \leq T_{1}
$$

then peak $\left(q_{3}^{(1)}(t)\right)=\operatorname{peak}\left(q_{2}^{(1)}(t)\right)\left(\right.$ and obviously peak $\left(q_{2}^{(1)}(t)\right)=\operatorname{peak}\left(q_{1}^{(1)}(t)\right)$ since (14) implies $\left.T_{2} \leq T_{1}\right)$, therefore the trajectory $q_{3}(t)$ reaches the maximum velocity $q_{\max }^{(1)}$. If, both conditions (13) and (14) are met, the velocity and the acceleration reach the maximum values $q_{\max }^{(i)}$, and $q_{3}(t)$ is a minimumtime trajectory. Conversely, when one (or both) of the two conditions is not true, the trajectory is compliant with the given bounds but it is not timeoptimal. In Fig. 4 the possible situations are exemplified. In the case (a) the 


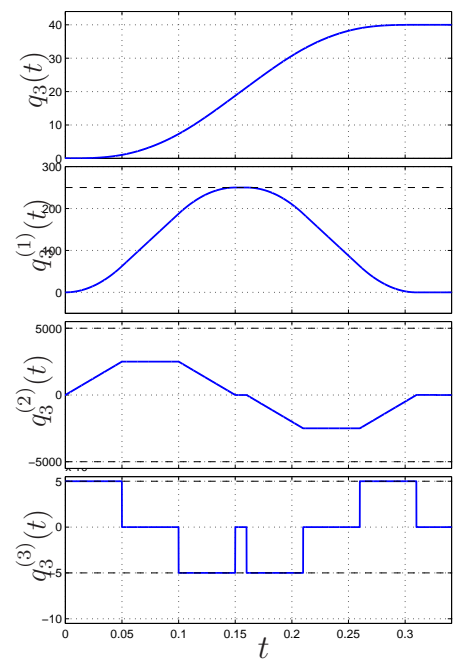

(a)

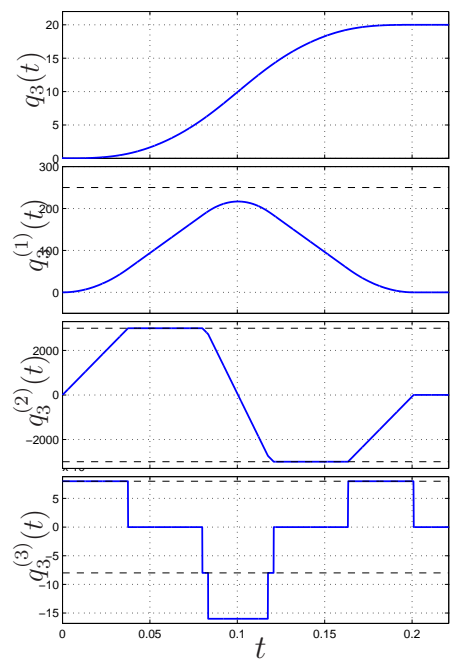

(b)

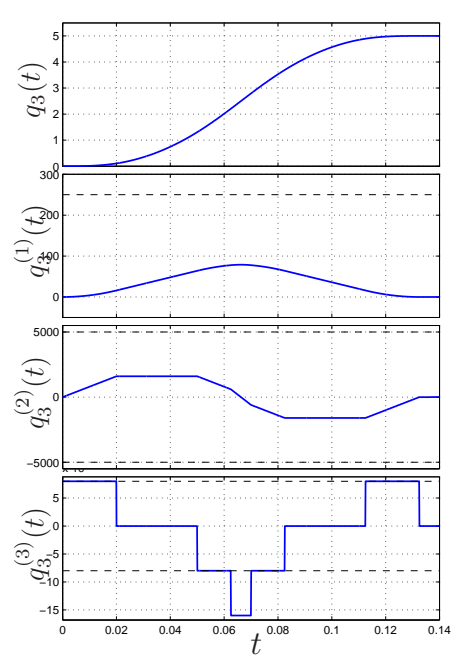

(c)

Figure 4: Double S velocity trajectories $q_{3}(t)$ obtained with $h=40 \mathrm{rad}, q_{\max }^{(1)}=250 \mathrm{rad} / \mathrm{s}$, $q_{\text {max }}^{(2)}=5000 \mathrm{rad} / \mathrm{s}^{2}, q_{\text {max }}^{(3)}=50000 \mathrm{rad} / \mathrm{s}^{3}(\mathrm{a}), h=20 \mathrm{rad}, q_{\text {max }}^{(1)}=250 \mathrm{rad} / \mathrm{s}, q_{\text {max }}^{(2)}=$ $3000 \mathrm{rad} / \mathrm{s}^{2}, q_{\text {max }}^{(3)}=80000 \mathrm{rad} / \mathrm{s}^{3}(\mathrm{~b}), h=5 \mathrm{rad}, q_{\max }^{(1)}=250 \mathrm{rad} / \mathrm{s}, q_{\max }^{(2)}=5000 \mathrm{rad} / \mathrm{s}^{2}$, $q_{\text {max }}^{(3)}=80000 \mathrm{rad} / \mathrm{s}^{3}(\mathrm{c})$.

time constants defining the three filters and computed on the basis of the given limit values, are

$$
T_{1}=0.16 \mathrm{~s}, \quad T_{2}=0.05 \mathrm{~s}, \quad T_{3}=0.1 \mathrm{~s},
$$

therefore (13) is false while (14) is true. Consequently, the maximum acceleration actually reached is not $q_{\max }^{(2)}=5000 \mathrm{rad} / \mathrm{s}^{2}$ but peak $\left(q_{3}^{(2)}(t)\right)=$ $2500 \mathrm{rad} / \mathrm{s}^{2}$. The case (b) is dual to (a). As a matter of fact

$$
T_{1}=0.08 \mathrm{~s}, \quad T_{2}=0.0833 \mathrm{~s}, \quad T_{3}=0.0375 \mathrm{~s},
$$

and therefore (13) is true and (14) is false. In this situation, the desired maximum acceleration is reached, while the maximum velocity is peak $\left(q_{3}^{(1)}(t)\right)=$ 


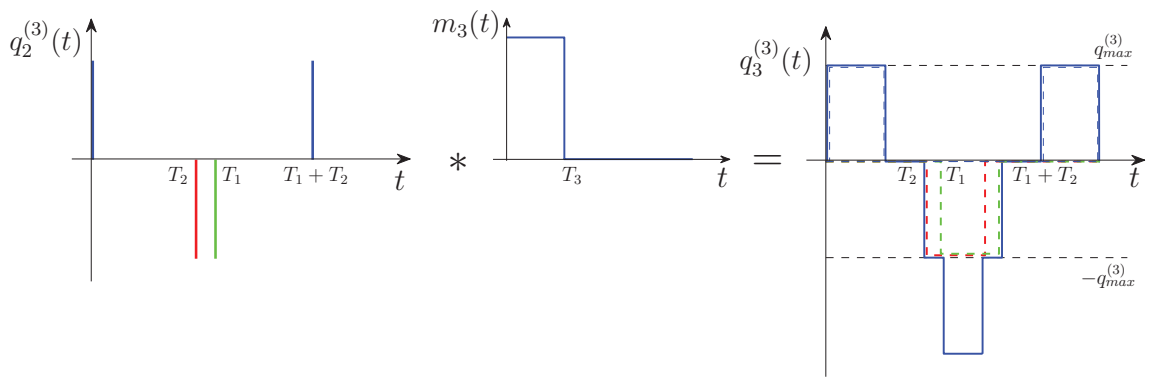

Figure 5: Jerk profile of a third order trajectory $q_{3}(t)$ characterized by the time constants $T_{i}, i=1,2,3$ with $T_{1}-T_{2}<T_{3}$.

$216.65<250 \mathrm{rad} / \mathrm{s}$. In the example reported in Fig. 4(c), that corresponds to

$$
T_{1}=0.02 \mathrm{~s}, \quad T_{2}=0.05 \mathrm{~s}, \quad T_{3}=0.0625 \mathrm{~s},
$$

both conditions (13) and (14) are not met and the trajectory does not reach neither the maximum velocity nor the maximum acceleration. Moreover, in the case $T_{1}<T_{2}+T_{3}$, that is in the examples of Fig. 4(b) and Fig. 4(c), the jerk exceeds the given bound. This is due to the fact that the jerk profile, obtained by convoluting a sequence of four impulsive functions by the impulse response of the third filter $m_{3}(t)$, is given by the superimposition of the single responses. Therefore if the distance between the application time-instants of two impulses with the same sign is smaller than the duration of $m_{3}(t)$, the responses are partially overlapped and produce a jerk profile that reaches a level twice the desired value, see Fig. 5.

The procedure shown so far can be iterated by adding further filters $M_{i}(s)$. In the general case, the expression of the minimum-time trajectory 
compliant with given constraints on the first $n$ derivatives, and therefore of order $n$, is

$$
q_{n}(t)=h u(t) * m_{1}(t) * \ldots * m_{n-1}(t) * m_{n}(t)
$$

or with a recursive formulation

$$
q_{n}(t)=q_{n-1}(t) * m_{n}(t)
$$

where $q_{0}(t)=h u(t)$. As already pointed out, the smoothness of the trajectory, that is the order of continuous derivatives, is strictly tied to the number of filters composing the chain. If one considers $n$ filters, the resulting trajectory will be of class $\mathcal{C}^{n-1}$. By increasing the smoothness of the trajectory, the duration augments as well. As a matter of fact the total duration of a trajectory planned by means of $n$ dynamic systems $M_{i}(s)$ is given by the sum of the lengths of the impulse response of each filter, i.e.

$$
T_{t o t}=T_{1}+T_{2}+\ldots+T_{n}
$$

The parameters $T_{i}$ can be set with the purpose of imposing desired bounds on velocity, acceleration, jerk and higher derivatives, i.e.

$$
\left|q_{n}^{(i)}(t)\right| \leq q_{\max }^{(i)}, \quad i=1, \ldots, n
$$

by assuming

$$
\begin{aligned}
& T_{1}=\frac{|h|}{q_{\max }^{(1)}} \\
& T_{i}=\frac{q_{\max }^{(i-1)}}{q_{\max }^{(i)}}, \quad i=2, \ldots, n
\end{aligned}
$$

with the constraints

$$
T_{j} \geq T_{j+1}+\ldots+T_{n}, \quad j=1, \ldots, n-1 .
$$


that guarantee that the trajectory, compliant with (17), is of minimum duration.

\subsection{Role of the constraints on $T_{i}$ and optimality of the trajectory}

The trajectory planner composed by $n$ filters $M_{i}(s)$ guarantees an output trajectory $q_{n}(t)$ compliant with the given constraints and of minimum duration if such constraints lead to time constants $T_{i}$ that verify (19). In this manner the $n$-th derivative of $q_{n}(t)$ is composed by $2^{n-1}$ distinct rectangular functions $m_{n}(t)$ properly shifted in time. The limit case occurs when two of these functions are contiguous (but not overlapped). Note that

$$
q_{n}^{(n)}(t)=q_{n-1}^{(n)}(t) * m_{n}(t)
$$

where (if the $n$ - 1-th trajectory is time optimal) $q_{n-1}^{(n)}$ is a train of $2^{n-1}$ impulses. The condition (19), that can be rewritten as

$$
T_{j}-T_{j+1}-\ldots-T_{n-1} \geq T_{n}, \quad j=1, \ldots, n-1,
$$

means that the distance between adjoining impulses composing $q_{n-1}^{(n)}$ must be greater than the duration $T_{n}$ of $m_{n}(t)$. In fact, $T_{j}-T_{j+1}-\ldots-T_{n-1}$, $j=1, \ldots, n-1$ represents the distances between pairs of impulses.

As already mentioned, if (19) is not true the trajectory $q_{n}(t)$, obtained as output of the cascade of $n$ filters, is not of minimum duration. In particular, if the inequality (19) is false for a given index $\hat{j}$, i.e.

$$
T_{\hat{j}}<T_{\hat{j}+1}+\ldots+T_{n},
$$

the trajectory is characterized by

$$
\operatorname{peak}\left(q_{n}^{(\hat{j})}(t)\right)<q_{\max }^{(\hat{j})}
$$


that is the limit value of the $\hat{j}$-th derivative is not reached. In this case, by reducing the value of $q_{\max }^{(\hat{j})}$ (that remains therefore compatible with the initial constraint) it is possible to increase $T_{\hat{j}}=q_{\max }^{(\hat{j}-1)} / q_{\max }^{(\hat{j})}$ until

$$
T_{\hat{j}}=T_{\hat{j}+1}+\ldots+T_{n}
$$

Note that, a reduction of $q_{\max }^{(\hat{j})}$ implies that $T_{\hat{j}+1}$ is also reduced and it may happens that (19) becomes false for $\hat{j}+1$.

In the general case in which inequalities (19) are false for $k$ values of the index $j$, namely $\hat{j}_{1}, \hat{j}_{2}, \ldots, \hat{j}_{k}$, it is necessary to reduce $q_{m a x}^{\left(\hat{j}_{i}\right)}, i=1, \ldots, k$ until

$$
T_{\hat{j}_{i}}=T_{\hat{j}_{i}+1}+\ldots+T_{n}, \quad i=1, \ldots, k
$$

and checking if (19) for the remaining index $j$ still holds true. In general, a closed-form solution of the problem cannot be found since many different situations may arise, but the cases $n=2$ and $n=3$ may be easily handled. In particular, for trapezoidal velocity trajectories generated by FIR filters $(n=2)$, when $T_{1}<T_{2}$, from $T_{1}=\frac{|h|}{q_{\max }^{(1)}}=\frac{q_{\max }^{(1)}}{q_{\max }^{(2)}}=T_{2}$, one may deduce the value of the maximum velocity that makes the trajectory time-optimal, that is

$$
\hat{q}_{\max }^{(1)}=\sqrt{|h| q_{\max }^{(2)}}<q_{\max }^{(1)} .
$$

The 2-nd order trajectory obtained with the same conditions of example in Fig. 3(b) but with the maximum velocity computed according to (23) is shown in Fig. 6, where it is compared with the original motion profile. Note that, the trajectory is still compliant with all the constraints, but it is considerably shorter than the motion profile obtained by simply applying (18) on the initial data (without modifying the maximum velocity). 


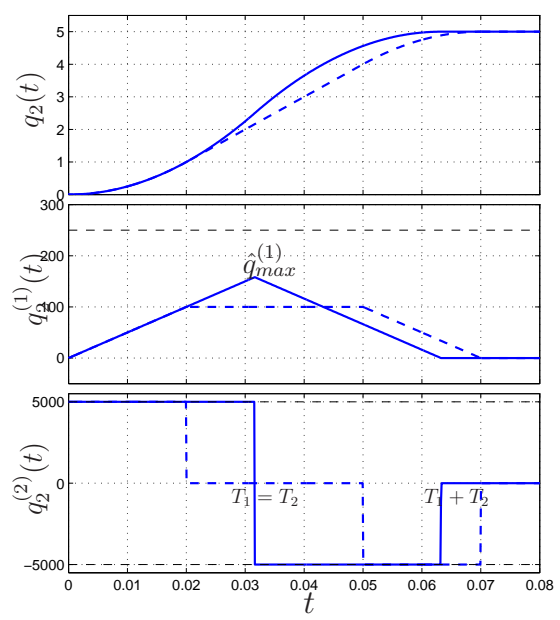

Figure 6: Trapezoidal velocity trajectory $q_{2}(t)$ obtained with the same conditions of example in Fig. $3(\mathrm{~b})$ but with the constraint $\hat{q}_{\max }^{(1)}=158.11 \mathrm{rad} / \mathrm{s}$ that leads to $T_{1}=T_{2}=$ $0.0316 \mathrm{~s}$.

In the case $n=3$, it may happen that

$$
T_{1}<T_{2}+T_{3} \Leftrightarrow \frac{|h|}{q_{\max }^{(1)}}<\frac{q_{\max }^{(1)}}{q_{\max }^{(2)}}+\frac{q_{\max }^{(2)}}{q_{\max }^{(3)}}
$$

or

$$
T_{2}<T_{3} \quad \Leftrightarrow \quad \frac{q_{\max }^{(1)}}{q_{\max }^{(2)}}<\frac{q_{\max }^{(2)}}{q_{\max }^{(3)}}
$$

and it is therefore necessary to modify the maximum value of the velocity or of the acceleration in order to make the two inequalities false. For instance, if (24) and (25) are both true, the optimal values of the maximum velocity and accelerations are

$$
\begin{aligned}
\hat{q}_{\max }^{(1)} & =\frac{|h|^{2 / 3} q_{\max }^{(3) 1 / 3}}{2^{2 / 3}} \\
\hat{q}_{\max }^{(2)} & =\frac{|h|^{1 / 3} q_{\max }^{(3)}{ }^{2 / 3}}{2^{1 / 3}} .
\end{aligned}
$$




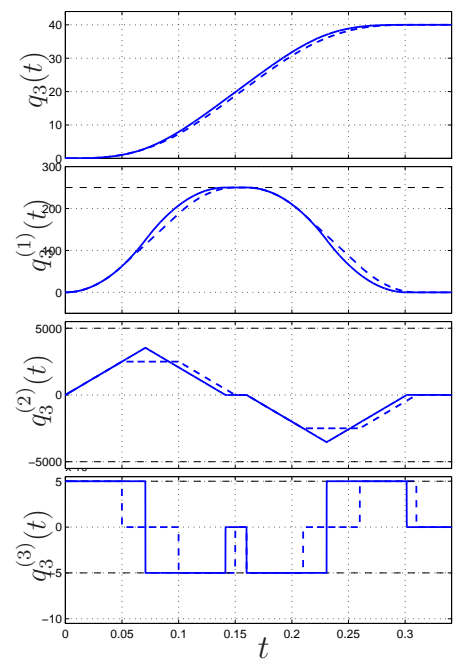

(a)

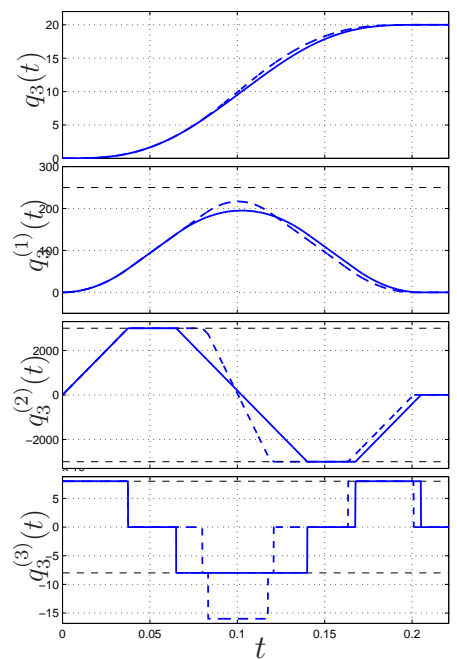

(b)

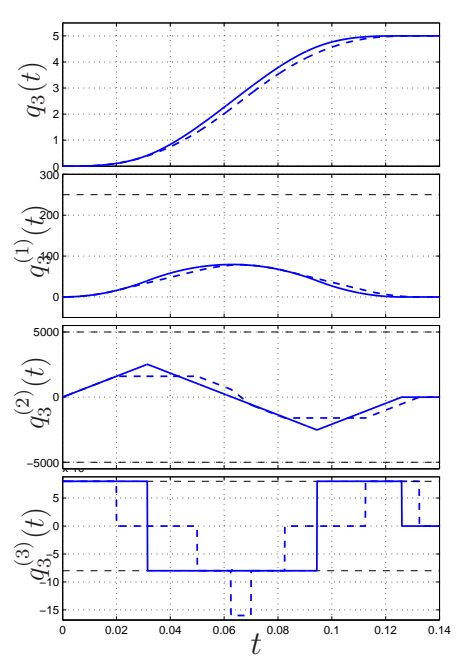

(c)

Figure 7: Double $\mathrm{S}$ velocity trajectories $q_{3}(t)$ obtained under the same constraints of examples in Fig. 4, but with $\hat{q}_{\max }^{(2)}=3535.5 \mathrm{rad} / \mathrm{s}^{2}$ (a), $\hat{q}_{\text {max }}^{(1)}=195.07 \mathrm{rad} / \mathrm{s}$ (b) and $\hat{q}_{\max }^{(1)}=79.37 \mathrm{rad} / \mathrm{s}, \hat{q}_{\max }^{(2)}=2519.8 \mathrm{rad} / \mathrm{s}^{2}(\mathrm{c})$.

If only (24) is valid

$$
\hat{q}_{\max }^{(1)}=\frac{1}{2}\left(-\frac{q_{\max }^{(2)^{2}}}{q_{\max }^{(3)}}+\sqrt{\frac{q_{\max }^{(2)}{ }_{q_{\max }^{(3)}}^{(3)}+4|h| q_{\max }^{(2)}}{}}\right)
$$

while, if only (25) holds true

$$
\hat{q}_{\max }^{(2)}=\sqrt{q_{\max }^{(1)} q_{\max }^{(3)}} .
$$

With these values used for the computation of parameters $T_{i}$, one obtains the minimum-time double $\mathrm{S}$ velocity trajectory compliant with the initial constraints. In Fig. 7, the trajectories of the examples reported in Fig. 4 are compared with those obtained by modifying the maximum velocity or acceleration according to the procedure above illustrated. In particular, the 
values of the filters parameters are

$$
T_{1}=0.16 \mathrm{~s}, \quad T_{2}=0.0707 \mathrm{~s}, \quad T_{3}=0.0707 \mathrm{~s}
$$

in case (a),

$$
T_{1}=0.1025 \mathrm{~s}, \quad T_{2}=0.0650 \mathrm{~s}, \quad T_{3}=0.375 \mathrm{~s}
$$

in case (b),

$$
T_{1}=0.063 \mathrm{~s}, \quad T_{2}=0.0315 \mathrm{~s}, \quad T_{3}=0.0315 \mathrm{~s}
$$

in case (c). Note that the duration of the trajectory in Fig. 7(b) is higher than the time-length of the original trajectory since the value of velocity provided by $(27)$, that is $\hat{q}_{\max }^{(1)}=195.07 \mathrm{rad} / \mathrm{s}$, is lower than the peak velocity of the initial trajectory, i.e. $\operatorname{peak}\left(q_{3}^{(1)}(t)\right)=216.65 \mathrm{rad} / \mathrm{s}$. In this case the modification of the time constants $T_{i}$ makes the trajectory a true double $\mathrm{S}$ trajectory, with the jerk $q^{(3)}(t) \in\left\{-q_{\max }^{(3)}, q_{\max }^{(3)}\right\}$, but it is worth noticing that in many applications the bound on the jerk is not due to physical limitations of the actuation system or of the load but it is a means to reduce oscillations and residual vibrations on the system. Therefore the limit values of jerk are a recommendation rather than a strict constraint. Moreover, as shown in Sec. 4.2, residual vibrations are related to the frequency spectrum of the trajectory (that depends on the parameters $T_{i}$ ) and not to the magnitude of jerk and other derivatives. For these reasons, in many cases a shorter duration of the trajectory may be preferable to a strict compliance with the bound on the jerk. 
In general, when the $\hat{j}$-th constraint on $T_{i}$, expressed by (19), is not met, one should act on $q_{\max }^{(\hat{j})}$. This modification leads to a reduction of the total duration of the trajectory only if the initial values of $T_{i}, i=\hat{j} \ldots n$ verify the inequality

$$
\left(T_{\hat{j}}-T_{\hat{j}-1}\right)^{2}>\left(T_{\hat{j}-2}+\ldots T_{n}\right)^{2} .
$$

Therefore, when (29) is not true it may be convenient to maintain the initial $T_{i}$, even if, strictly speaking, the resulting profile cannot be considered an optimal multi-segment trajectory, according to the usual definition. Consider that, although (19) is false, the bounds on $q_{\max }^{(i)}, i=1, \ldots, n-1$ are met in any case and only $q_{\max }^{(n)}$ is overcome.

\subsection{Derivatives of a generic trajectory}

A trajectory generator should provide not only the position profile of the trajectory but also the related profiles of velocity, acceleration, jerk, etc. which are needed to implement e.g. feed-forward actions on the controlled system.

The computation of the derivatives of a trajectory of generic order $n$, that is obtained by a cascade of $n$ filters, is straightforward by considering the definition (15) and the property of convolution product (2). In fact,

$$
\begin{aligned}
q_{n}^{(1)}(t) & =q_{n-1}(t) * m_{n}^{(1)}(t) \\
& =q_{n-1}(t) * \frac{1}{T_{n}}\left(\delta(t)-\delta\left(t-T_{n}\right)\right) \\
& =\frac{1}{T_{n}}\left(q_{n-1}(t)-q_{n-1}\left(t-T_{n}\right)\right) .
\end{aligned}
$$




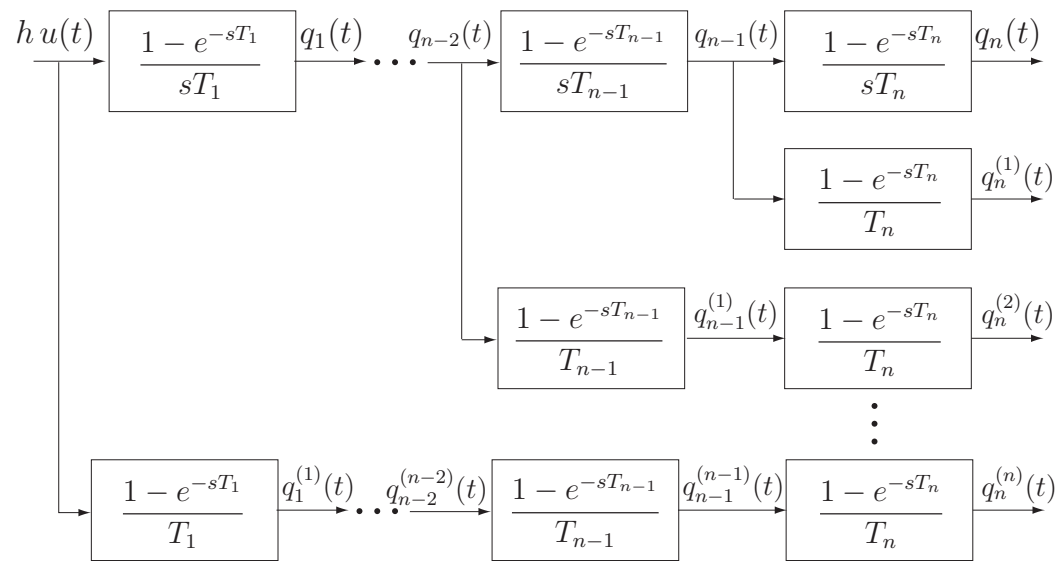

Figure 8: System composed by $n$ filters for the computation of an optimal trajectory of class $\mathcal{C}^{n-1}$ and of all the derivatives of order $i=1, \ldots, n$.

The generic derivative of $k$-th order, can be calculated in a recursive manner as

$$
q_{n}^{(k)}(t)=\frac{1}{T_{n}}\left(q_{n-1}^{(k-1)}(t)-q_{n-1}^{(k-1)}\left(t-T_{n}\right)\right)
$$

with $q_{n-k}^{(0)}(t)=q_{n-k}(t)$. Figure 8 shows the block-scheme representation of the filter for the computation of the trajectory and its derivatives, obtained by iterating and Laplace transforming (31). Note that the filter of Fig. 8 gives a closed form expression (in terms of Laplace transform) of the derivatives and does not simply provide their numerical value.

\subsection{Frequency characterization of the trajectory/filter}

The spectrum of the trajectory can be readily deduced by considering its expression in terms of Laplace transform (directly obtained from (15)), i.e.

$$
Q_{n}(s)=\frac{h}{s} \cdot M_{1}(s) \cdot M_{2}(s) \cdot \ldots \cdot M_{n}(s) .
$$


As a matter of fact, as it is well known, the Fourier transform of $q_{n}(t)$ immediately descends from $Q_{n}(s)$, being the restriction to the imaginary axis, i.e. $Q_{n}(j \omega)$. Therefore, the closed form expression of $Q_{n}(j \omega)$ is given by the products of the Fourier signal corresponding to the input $h u(t)$ and of the frequency responses of the filters composing the trajectory generator:

$$
Q_{n}(j \omega)=\frac{h}{j \omega} \cdot M_{1}(j \omega) \cdot M_{2}(j \omega) \cdot \ldots \cdot M_{n}(j \omega)
$$

where

$$
\begin{aligned}
M_{i}(j \omega) & =\frac{1}{T_{i}} \frac{1-e^{j \omega T_{i}}}{j \omega} \\
& =e^{-j \frac{\omega T_{i}}{2}} \frac{\sin \left(\frac{\omega T_{i}}{2}\right)}{\frac{\omega T_{i}}{2}} .
\end{aligned}
$$

Since the frequency characterization of the trajectories, including their derivatives and, in particular, the acceleration, is a useful tool to predict vibratory phenomena in the systems to which the trajectories are applied (Biagiotti and Melchiorri, 2008), it is necessary to obtain the expression of the spectrum of the generic $k$-th derivative of $q_{n}(t)$. Because of the properties of Laplace transforms, this result is straightforward. As a matter of fact, the Laplace transform of $q_{n}^{(k)}(t)$ is given by

$$
Q_{n}^{(k)}(s)=s^{k} Q_{n}(s)
$$

and therefore the expression of the spectrum of $q_{n}^{(k)}(t)$ is

$$
\begin{aligned}
Q_{n}^{(k)}(j \omega) & =(j \omega)^{k} \cdot Q_{n}(j \omega) \\
& =h \cdot(j \omega)^{k-1} \cdot M_{1}(j \omega) \cdot M_{2}(j \omega) \cdot \ldots \cdot M_{n}(j \omega) .
\end{aligned}
$$

In conclusion, the amplitude spectrum of $q_{n}(t)$ and its derivatives, i.e. $\left|Q_{n}^{(k)}(j \omega)\right|$, is given by the product of two main elements: 


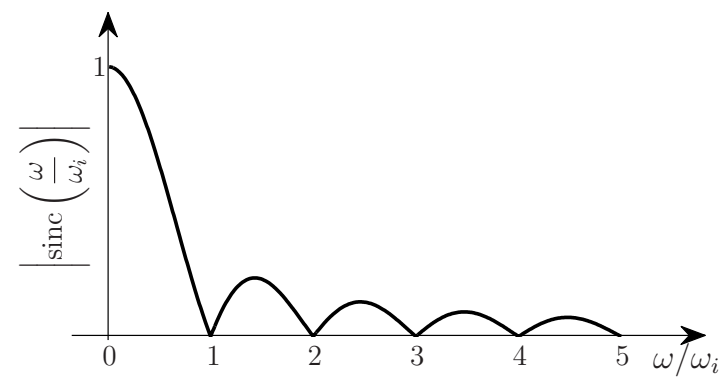

Figure 9: Magnitude of the frequency response of the filter $M_{i}(s)$.

- a power of $\omega$, i.e. $\omega^{k-1}$, being $k$ the order of the derivative;

- the (magnitude of the) frequency response of the chain of $n$ filters $M_{i}(s)$.

The frequency response of the cascade of filters is the product of the single frequency responses $M_{i}(j \omega), i=1, \ldots, n$, whose magnitude is

$$
\left|M_{i}(j \omega)\right|=\left|\frac{\sin \left(\frac{\omega T_{i}}{2}\right)}{\frac{\omega T_{i}}{2}}\right|=\left|\operatorname{sinc}\left(\frac{\omega}{\omega_{i}}\right)\right|
$$

where $\operatorname{sinc}(\cdot)$ denotes the normalized sinc function defined as $\operatorname{sinc}(x)=\frac{\sin (\pi x)}{\pi x}$ and $\omega_{i}=\frac{2 \pi}{T_{i}}$. Note that the function $\left|M_{i}(j \omega)\right|$, shown in Fig. 9, is equal to zero for $\omega=k \omega_{i}$, with $k$ integer. This property can be profitably exploited to properly choose the parameters of the trajctory/filter with the purpose of nullifying the spectrum of the trajectory at critical frequencies, for instance the eigenfrequencies of the plant. For this aim, if $\omega_{r}$ denotes a resonant frequency, it is sufficient to assume

$$
\omega_{i}=\frac{\omega_{r}}{l} \Leftrightarrow T_{i}=l \frac{2 \pi}{\omega_{r}}, \quad l=1,2, \ldots
$$


This result generalizes what has been presented in Olabi et al. (2010) where, with reference to a double $\mathrm{S}$ velocity trajectory, it is recognized that in order to suppress residual vibrations due to the dominating vibratory mode of an axis of motion it is necessary to assume the duration of the "jerk period" (in which the jerk remains constant) equal to a multiple of the natural period of the vibrational mode. According to (34) the reduction of residual vibrations caused by resonant frequencies of the plant can be achieved with multi-segment trajectories of any order provided that the time constant $T_{i}$ of a filter $M_{i}(s)$ is $l$ times, $l$ integer, the dominating natural period $\frac{2 \pi}{\omega_{r}}$.

\section{Discretization of trajectories and FIR filters}

The expression of a generic trajectory is usually provided in the continuoustime domain by means of an analytic function of time $t$. On the other hand, for being used as a reference signal for a computer controlled system, it needs to be evaluated at discrete-time instants $t_{k}=k T_{s}$, being $T_{s}$ the sampling period. For this reason, it is convenient to directly express the trajectory in the discrete-time domain, obtaining a filter able to provide at each time instant $k T_{s}$ the value $q_{n}(k)$.

Starting from the expression of the continuous trajectory planner, obtained by connecting $n$ filters $M_{i}(s)$ in a cascade configuration fed by a step function, it is possible to deduce an equivalent discrete-time system by discretizing the filters with one of the techniques available in the literature (Franklin et al., 1998) and providing as input the sequence obtained by sampling with a pe$\operatorname{riod} T_{s}$ the continuous step function. In particular, the adoption of backward differences method leads to a discrete-time system composed by a chain of 
FIR filters, whose transfer function results

$$
\begin{aligned}
M_{i}(z) & =\left.M_{i}(s)\right|_{s=\frac{1-z^{-1}}{T_{s}}} \\
& =\frac{T_{s}}{T_{i}} \frac{1-z^{-N_{i}}}{1-z^{-1}} \\
& =\frac{1}{N_{i}} \frac{1-z^{-N_{i}}}{1-z^{-1}}
\end{aligned}
$$

where

$$
N_{i}=\frac{T_{i}}{T_{s}}
$$

is the number of samples (not null) of the filter response, which is also equal to the number of elements composing the FIR filter (usually called taps) as they appear in the equivalent (nonrecursive) formulation

$$
M_{i}(z)=\frac{1}{N_{i}}+\frac{1}{N_{i}} z^{-1}+\frac{1}{N_{i}} z^{-2}+\ldots+\frac{1}{N_{i}} z^{-N_{i}-1} .
$$

Note that (37) is the expression of a moving average filter, which averages the last $N_{i}$ samples.

Finally, the expression of $Q_{n}(z)$ representing the discrete-time trajectory $q_{n}(k)$ in the Z-domain results

$$
Q_{n}(z)=\frac{h}{1-z^{-1}} \cdot M_{1}(z) \cdot M_{2}(z) \cdot \ldots \cdot M_{n}(z)
$$

where $\frac{1}{1-z^{-1}}$ is the Z-transform of

$$
u(k)= \begin{cases}1, & \text { for } k=0,1,2, \ldots \\ 0, & \text { for } k<0\end{cases}
$$

It is worth highlighting that the temporal sequence $q_{n}(k)=\mathcal{Z}^{-1}\left\{Q_{n}(z)\right\}$ only approximates the corresponding continuous-time trajectory $q_{n}(t)$, as shown 


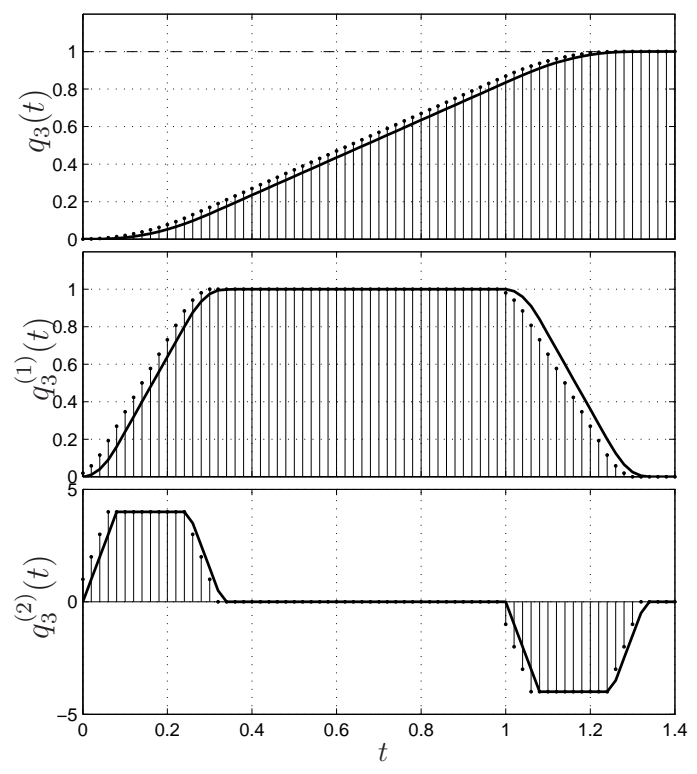

Figure 10: Comparison between the output of the third order continuous-time filter (solid line) and that of corresponding cascade of FIR filters (dots) obtained by discretization $\left(T_{s}=0.02\right)$.

in Fig. 10 where the samples of $q_{3}(k)$ are compared with the profile of the corresponding third order trajectory $q_{3}(t)$. In this case, the sampling period has been intentionally assumed quite large if compared with the total duration of the trajectory $\left(T_{s}=0.02 \mathrm{~s}\right)$ in order to point out the approximation error. However, it is possible to prove that when $T_{s}$ goes to zero, such an error vanishes. From a practical point of view, this means that, for sufficiently small sampling periods, the sequence $q_{n}(k)$ can be used in lieu of the corresponding function $q_{n}(t)$ without appreciable differences. The bank of $n$ FIR filters shown in Fig. 11, fed with sampled step functions (defining the desired final positions), can be therefore adopted to generate the trajectory of order $n$. 
An approximation exists also in the frequency domain between the spectra of $Q_{n}(z)$ and of $Q_{n}(j \omega)$. As a matter of fact

$$
\begin{aligned}
Q_{n}\left(e^{j \omega T_{s}}\right) & =\frac{h}{1-e^{-j \omega T_{s}}} M_{1}\left(e^{j \omega T_{s}}\right) M_{2}\left(e^{j \omega T_{s}}\right) \ldots M_{n}\left(e^{j \omega T_{s}}\right) \\
& \approx \frac{h}{j \omega T_{s}} M_{1}\left(e^{j \omega T_{s}}\right) M_{2}\left(e^{j \omega T_{s}}\right) \ldots M_{n}\left(e^{j \omega T_{s}}\right)
\end{aligned}
$$

with

$$
\begin{aligned}
M_{i}\left(e^{j \omega T_{s}}\right) & =\frac{1}{N_{i}} \frac{1-e^{-j N_{i} \omega T_{s}}}{1-e^{-j \omega T_{s}}} \\
& \approx \frac{1}{N_{i}} \frac{1-e^{-j \omega T_{i}}}{j \omega T_{s}} \\
& =\frac{1}{T_{i}} \frac{1-e^{-j \omega T_{i}}}{j \omega}
\end{aligned}
$$

where the Taylor series expansion of exponential function truncated at the first order, i.e. $e^{-j \omega T_{s}} \approx 1-j \omega T_{s}$ has been used. Note that because of this approximation, (38) and (39) are true only if $\omega T_{s}$ is small enough. As a consequence the smaller the sampling time $T_{s}$ is, the wider the frequency range of validity of (38) will be. Within this range, the considerations of Sec. 2.3 remain valid and therefore the desired trajectory $q_{n}(t)$ can be planned by means of the chain of discrete-time filters, whose characteristic parameters $N_{i}$ are directly related to the periods $T_{i}$ defining the trajectory by means of (36).

$$
\stackrel{h u(k)}{\longrightarrow} \frac{1}{N_{1}} \frac{1-z^{-N_{1}}}{1-z^{-1}} \stackrel{q_{1}(k)}{\longrightarrow} \frac{1}{N_{2}} \frac{1-z^{-N_{2}}}{1-z^{-1}} \stackrel{q_{2}(k)}{\longrightarrow} \stackrel{q_{n-1}(k)}{\longrightarrow} \frac{1}{N_{n}} \frac{1-z^{-N_{n}}}{1-z^{-1}} \stackrel{q_{n}(k)}{\longrightarrow}
$$

Figure 11: System composed by $n$ moving average filters for the computation of an optimal trajectory of class $\mathcal{C}^{n-1}$ at discrete time-instants $k T_{s}$. 
Finally, it is worth noticing that the structure proposed in Fig. 11 for the generation of time-optimal trajectories results very efficient from a computational point of view. As a matter of fact, the $i$-th FIR filter is characterized by the differences equation

$$
q_{i}(k)=q_{i}(k-1)+\frac{1}{N_{i}}\left(q_{i-1}(k)-q_{i-1}\left(k-N_{i}\right)\right), \quad i=1, \ldots, n
$$

and, for the evaluation of $q_{i}$ at the $k$-th sampling instant, only two additions and one multiplication are necessary. Therefore the trajectory of order $n$ requires $n$ multiplications and $2 n$ additions. Note that the general expression of multi-segment trajectories based on polynomials is

$$
q_{n}(t)=a_{i, n} t^{n}+a_{i, n-1} t^{i, n-1}+a_{i, 1} t+a_{i, 0}, \quad i=1, \ldots, 2^{n}-1
$$

where the coefficients $a_{i, j}$ must be properly computed for each of the $2^{n}-1$ tracts composing the motion profile. Obviously some of these coefficients are null in specific segments, but in the worst case an efficient evaluation ${ }^{2}$ of the trajectory for a given value of $t$ needs at least $n$ multiplications and $n$ additions. Therefore, the order of complexity of the chain of FIR filters and of the equivalent polynomial expression is comparable, but in case of direct evaluation of the analytic expression of the trajectory it is also necessary a search algorithm to determine which segment must be considered at a specific value of time $t$ and a switch statement to apply a different expression for each tract. For this reason, especially for high values of the order $n$, the expression based on FIR filters may be preferable to the standard analytic expression of

\footnotetext{
${ }^{2}$ For the estimation of the complexity of the polynomial evaluation the so-called Horner formula has been assumed.
} 
multi-segment trajectories both in terms of implementation complexity and computational cost.

\section{Case Studies}

The proposed trajectory generator, composed by $n$ running average filters, presents two main features which make it very attractive, namely

- the possibility of planning multi-segment trajectories online simply by changing the input signal, composed by elementary step functions;

- the clear frequency characterization of the trajectory obtained as output of the filters chain, which makes it possible to choose the value of the characteristic parameter $T_{i}$ of each filter on the basis of the desired trajectory spectrum.

These features are now exploited to define tasks that would require complicated procedures with the analytic expression of multi-segment trajectories, while are immediate with FIR filters.

\subsection{Multi-point time-optimal trajectories}

Complex trajectories composed by several segments joining a set of viapoints $p_{j}, j=0, \ldots, m$ can be planned online by feeding the discrete-time filters of Fig. 11 with a staircase function, whose constant values are the desired (final) positions $p_{j}$ :

$$
\boldsymbol{p}(t)=\sum_{j=1}^{m} p_{j} u\left(t-t_{j}\right)
$$

where $t_{j}$ is the starting time-instant of the $j$-th tract. Note that, in order to assure the compliance with the given bounds, a new tract cannot start 

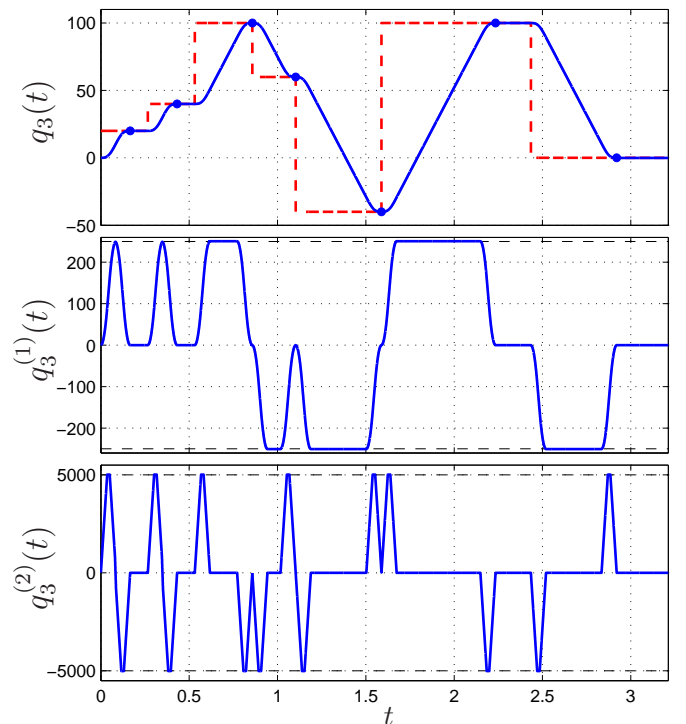

(a)

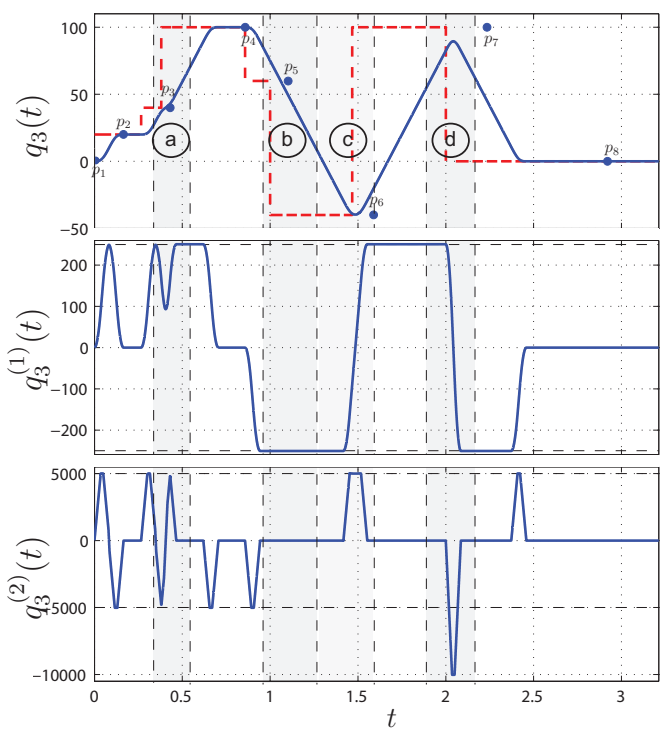

(b)

Figure 12: Complex motion obtained with a third order trajectory passing through a sequence of via-points $\boldsymbol{p}=\{0,20,40,100,60,-40,100,0\}$, with the constraints $q_{\max }^{(1)}=$ $250 \mathrm{rad} / \mathrm{s}, q_{\text {max }}^{(2)}=5000 \mathrm{rad} / \mathrm{s}^{2}, q_{\text {max }}^{(3)}=140000 \mathrm{rad} / \mathrm{s}^{3}$. In case (a) initial time instants $t_{j}$ meet the condition (41), while in (b) such a condition is violated.

before the previous one has ended. For a generic planer of order $n$, this consideration can be translated into a constraint on the initial time-instants $t_{j}$, i.e.

$$
t_{j} \geq t_{j-1}+T_{1}+T_{2}+\ldots+T_{n-1}+T_{n}, \quad j=1, \ldots, m .
$$

In this way, the trajectory is composed by a sequence of point to point motions that start/end with null velocity, acceleration, jerk, etc. In Fig. 12(a) a motion profile composed by a sequence of third order trajectories $q_{3}(t)$ (double $\mathrm{S}$ velocity trajectories) is shown. The trajectory generator is composed in this case by three running average filters, with sampling time $T_{s}=0.0001$ 
s, defined by the time constants

$$
\begin{aligned}
T_{1, j} & =\frac{\left|h_{j}\right|}{q_{\max }^{(1)}}=\frac{\left|h_{j}\right|}{15} \mathrm{~s} \\
T_{2} & =\frac{q_{\max }^{(1)}}{q_{\max }^{(2)}}=0.05 \mathrm{~s} \rightarrow N_{2}=\frac{0.05}{0.0001}=500 \\
T_{3} & =\frac{q_{\max }^{(2)}}{q_{\max }^{(3)}}=0.035 \mathrm{~s} \rightarrow N_{3}=\frac{0.035}{0.0001}=350
\end{aligned}
$$

where $h_{j}=p_{j}-p_{j-1}, j=1, \ldots, m$ represents the displacement of the $j$-th tract. Note that the limit values of velocity, acceleration, jerk, are generally constant and therefore $N_{2}$ and $N_{3}$ are the same for all tracts and are fixed before the motion starts. Conversely, in order to guarantee a constant velocity with different displacements $h_{j}$, it is necessary to change online the structure of the first FIR filter of the trajectory planner. In particular, it is needed to adapt the number of taps of the filter to the desired displacement value $h_{j}$, according to the formula $N_{1, j}=\operatorname{round}\left(\frac{\left|h_{j}\right|}{T_{s} \cdot q_{\max }^{(1)}}\right)$. Obviously, this operation must be performed whenever the input signal defining the final position changes (and therefore a new displacement $h_{j}$ is required) and it must guarantee the continuity of the trajectory and of its derivatives up to the order $n-1$. Since a new via-point is provided only when the previous one has been reached, when the input changes all the FIR filters (and in particular the first one) are in a steady-state condition. For the first filter fed with constant signals, this means that both the output and all the internal states are equal to the input value. As a consequence, when additional taps are added to $M_{1}(z)$, in order to keep the output unchanged, it is necessary to set the values of the new internal states equal to those of the existing states. When some taps are eliminated, the values of the remaining internal states 
are not modified.

As above mentioned, the trajectory planned with the proposed generator is composed by rest to rest motion segments between the via-points and it is not possible to specify desired values of velocity, acceleration, jerk, etc. different from zero at these points. However, it is worth noticing that by modifying (online) the staircase function $\boldsymbol{p}(t)$, the output trajectory does not stop at the via-points. In Fig. 12(b) different situations that may occur are illustrated. In cases (a) and (b) the required displacements of two consecutive tracts have the same sign while in (c) and (d) they are opposite. In particular, in the tract denoted by (a), the level corresponding to the via-point $p_{4}$ is provided before $p_{3}$ is reached. As a consequence the trajectory crosses $p_{3}$ without stopping on it. Note that the velocity firstly decreases and then increases without becoming zero. In the segment (b), the point $p_{6}$ is given before the trajectory starts decelerating. In this manner, the velocity remains constant at the maximum value. In the segment (c), the input function $\boldsymbol{p}(t)$ is modified when the acceleration of the previous tract starts decreasing, that is at time $T_{\text {tot }}-T_{3}$ being $T_{t o t}$ the total duration of the motion law between $p_{5}$ and $p_{6}$. In this way the deceleration of the former segment and the acceleration of the latter one, that have the same sign, are superimposed but the two contributions are compensated each other and the acceleration profile of the resulting trajectory does not overcome the limit value. A different situation arises in case $(\mathrm{d})$, where the next via-point $p_{7}$ is provided (and therefore the trajectory segment between $p_{6}$ and $p_{7}$ begins) before the acceleration of the previous tract starts decreasing. As a consequence, the deceleration and the acceleration of the two segments, that also in this case have the same sign, 
are superimposed and lead to a total acceleration that reaches a peak value twice the desired bound.

From this analysis, it comes out that the condition (41), that guarantees that all the segments composing the trajectory are point to point movements with initial and final derivatives null, is too conservative with respect to the problem of the compliance with the limits of velocity, acceleration, jerk, etc. As a matter of fact, only when two consecutive tracts are obtained for displacements with opposite signs the bounds are violated if a new reference input is provided (more than $T_{3}$ seconds) before the end of the former segment. In this case, it is convenient to wait that the trajectory stops at a given via-point before providing the next reference point. Conversely, if the next via-point requires a movement in the same direction of the current trajectory, it can be given to the chain of filters at any time. Clearly, in order to allows a "smooth" modification of the first FIR filter according to the desired displacement, it is necessary to wait at least $T_{1}$ seconds from the last via-point.

Another possible design strategy for the trajectory planner consists in assuming a constant value for $N_{1}$. For instance, if the required displacement $h_{j}$ are known in advance, one may choose

$$
T_{1}=\frac{\max _{j}\left(\left|h_{j}\right|\right)}{q_{\max }^{(1)}} \rightarrow N_{1}=\operatorname{round}\left(\frac{T_{1}}{T_{s}}\right)
$$

in order to guarantee that the maximum velocity is never exceeded. In this way the duration of the trajectory is constant, whatever the displacement $h_{j}$ may be. This property can be exploited to synchronize the motions among different axes, as shown in Fig. 13 where the trajectories passing through two 


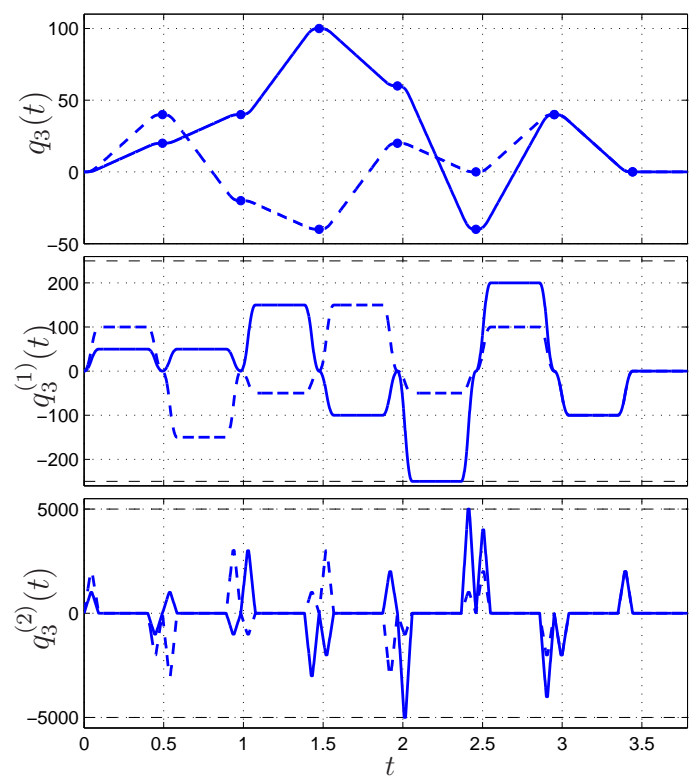

Figure 13: Synchronization of two complex motions composed by third order trajectories passing through $\boldsymbol{p}_{1}=\{0,20,40,100,60,-40,40,0\}$ and $\boldsymbol{p}_{2}=$ $\{0,40,-20,-40,20,0,40,0\}$ respectively. The constraints are the same of the example in Fig. 12.

different sets of via-points, and obtained with two chains of filters characterized by the same parameters $T_{i}$, are reported. Note that the two motion profiles are not time-optimal, in the sense that the maximum velocity and acceleration are reached only in a segment, but the corresponding tracts of the two profiles are perfectly synchronized, that is they start/end at the same instants. Moreover, also the acceleration/deceleration phases and the constant velocity phases within the corresponding tracts of the two trajectories are synchronized. 


\subsection{Multi-segment trajectories with frequency specifications}

In the previous example the parameters of the trajectory generator are obtained on the basis of constraints (velocity, acceleration, jerk) expressed in the time-domain. On the other hand, as already mentioned, it is also possible to take into account frequency constraints, that may arise because of critical frequencies of the plant that tracks this motion profile. Therefore, it is possible to combine the advantages of time-optimal multi-segment trajectories with those of the approaches that filter the input trajectories to properly shape their spectrum, see Singer et al. (1999) for a comprehensive overview on this argument.

Let us consider the standard motion system shown in Fig. 14, composed by two inertias with an elastic transmission lightly damped (Lambrechts et al., 2005; Barre et al., 2005; Meckl and Arestides, 1998), whose model (from the motor position $q_{m}$ to the load position $q_{l}$ ) can be described by the transfer function

$$
G_{m l}(s)=\frac{Q_{l}(s)}{Q_{m}(s)}=\frac{2 \delta \omega_{n} s+\omega_{n}^{2}}{s^{2}+2 \delta \omega_{n} s+\omega_{n}^{2}}
$$

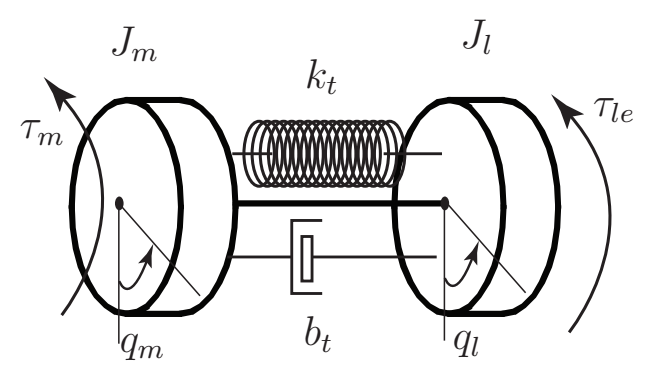

Figure 14: Lumped constant model of a motion system with elastic transmission. 


\begin{tabular}{llll}
\hline Parameter & Symbol & Value & Unit \\
\hline Motor inertia & $J_{m}$ & $0.72 \times 10^{-5}$ & $\mathrm{~kg} \mathrm{~m}^{2}$ \\
Load inertia & $J_{l}$ & $0.23 \times 10^{-5}$ & $\mathrm{~kg} \mathrm{~m}^{2}$ \\
Spring stiffness & $k_{t}$ & 0.156 & $\mathrm{~N} \mathrm{~m}$ \\
Internal damping & $b_{t}$ & $1.0 \times 10^{-5}$ & $\mathrm{~N} \mathrm{~m} \mathrm{~s}$ \\
\hline
\end{tabular}

Table 1: Motion system parameters.

with

$$
\omega_{n}=\sqrt{\frac{k_{t}}{J_{l}}}, \quad \delta=\frac{b_{t}}{2 \sqrt{k_{t} J_{l}}} .
$$

The parameters of the system, reported in Tab. 1, are derived from Lambrechts et al. (2005), as well as the trajectory constraints $\left(q_{\max }^{(1)}=250 \mathrm{rad} / \mathrm{s}\right.$, $\left.q_{\max }^{(2)}=5000 \mathrm{rad} / \mathrm{s}^{2}, q_{\max }^{(3)}=5 \times 10^{5} \mathrm{rad} / \mathrm{s}^{3}\right)$. The resonant frequency of the system results $\omega_{r} \approx \omega_{n}=260.43 \mathrm{rad} / \mathrm{s}$, while $\delta=0.0083$.

By supposing that an ideal control system imposes to the motor $\left(J_{m}\right)$ the desired motion profile, that is $q_{m}(t)=q_{r e f}(t)$, being $q_{r e f}(t)$ a trajectory obtained with the generator proposed in previous sections, it is possible to analyze the effects of a particular choice of the trajectory parameters on the dynamic behavior of the plant and in particular on the tracking error, defined as $\varepsilon(t)=q_{\text {ref }}(t)-q_{l}(t)=q_{m}(t)-q_{l}(t)$. Obviously, the choice of the parameters of the filter is critical only when the spectral components of the trajectory are appreciable in the neighborhood of the eigenfrequency of the plant. If this occurs, a design of the trajectory that neglects the dynamic characteristics of the plant may lead to large tracking errors during the motion, and to residual oscillations when the motion stops. For instance, the response of $G_{m l}(s)$ to a trapezoidal velocity trajectory obtained by assuming 


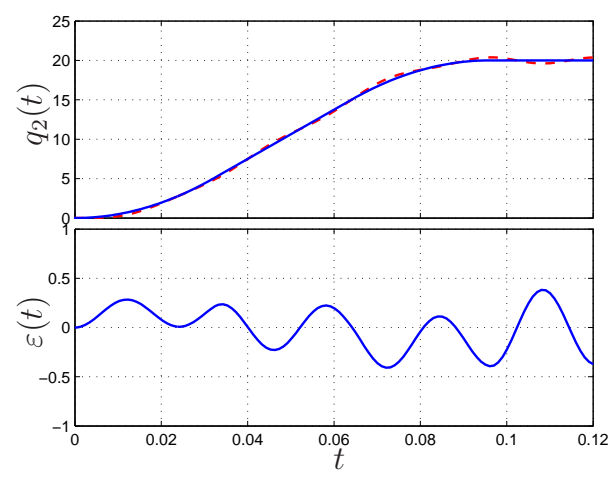

(a)

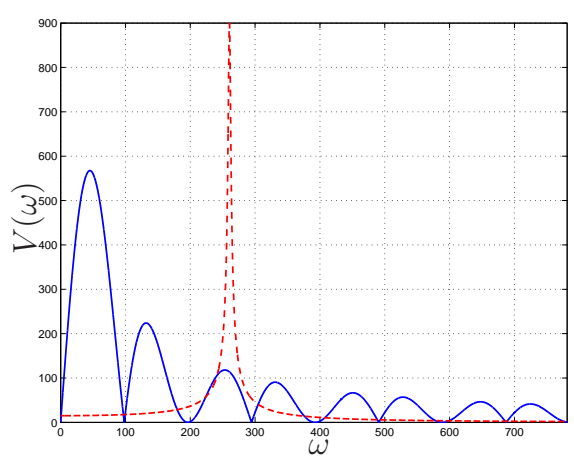

(b)

Figure 15: Response of the elastic system $G_{m l}(s)$ to a trapezoidal velocity trajectory obtained with $T_{1}=0.064 \mathrm{~s}$ and $T_{2}=0.032 \mathrm{~s}$ : tracking error (a) and frequency spectrum of the acceleration (b).

$T_{1}=0.064 \mathrm{~s}$ and $T_{2}=0.032 \mathrm{~s}\left(T_{t o t}=0.096 \mathrm{~s}\right)$ is shown in Fig. 15(a) along with the error $\varepsilon(t)$. Note the considerable value of the error at the end of motion, in particular if compared with the error obtained by applying to the system the trapezoidal velocity trajectory of Fig. 16, characterized by the same total duration $T_{\text {tot }}=0.096 \mathrm{~s}$, but obtained with $T_{1}=3 T_{0}$ and $T_{2}=T_{0}$ (being $T_{0}=\frac{2 \pi}{\omega_{r}}=0.0242 \mathrm{~s}$ the natural period of $G_{m l}(s)$ ). By analyzing the spectral contents of the two trajectories, and in particular of the acceleration profiles, it is possible to explain such results. The dynamic relation between the reference trajectory $q_{r e f}(t)$ and the tracking error $\varepsilon$, obtained from (42) after simple algebraic manipulations, is given by

$$
\frac{E(s)}{Q_{r e f}(s)}=\frac{s^{2}}{s^{2}+2 \delta \omega_{n} s+\omega_{n}^{2}}
$$




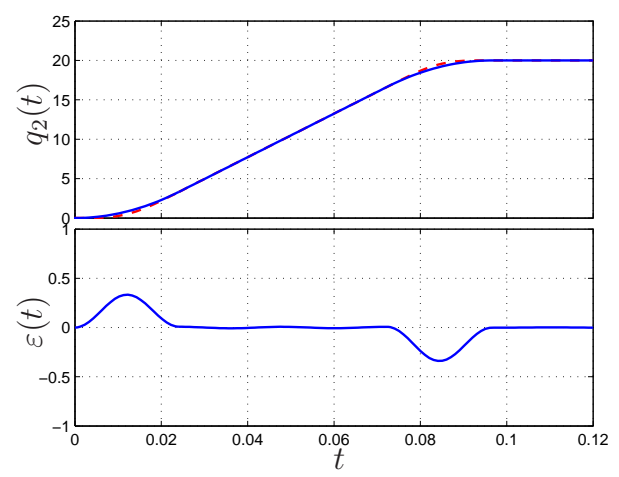

(a)

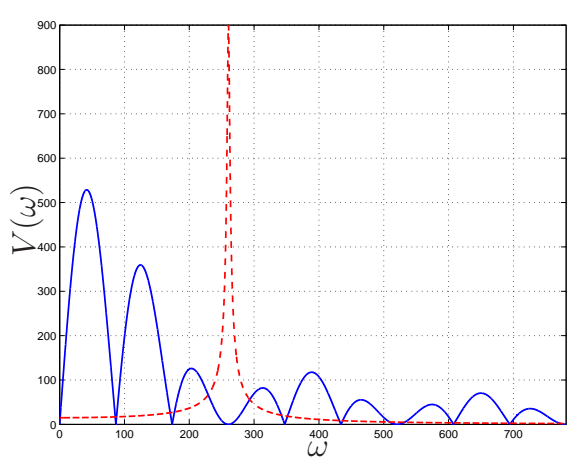

(b)

Figure 16: Response of the elastic system $G_{m l}(s)$ to a trapezoidal velocity trajectory obtained with $T_{1}=3 T_{0}$ and $T_{2}=T_{0}$ : tracking error (a) and frequency spectrum of the acceleration (b).

where $E(s)=\mathcal{L}\{\varepsilon(t)\}$ and $Q_{\text {ref }}(s)=\mathcal{L}\left\{q_{\text {ref }}(t)\right\}$. Note that (43) can be rewritten as

$$
\frac{E(s)}{Q_{r e f}^{(2)}(s)}=\frac{E(s)}{s^{2} Q_{r e f}(s)}=\frac{1}{s^{2}+2 \delta \omega_{n} s+\omega_{n}^{2}}=G_{\varepsilon}(s)
$$

that is $\varepsilon$ can be obtained by applying the second derivative of the reference signal $q_{r e f}(t)=q_{n}(t)$, i.e. the acceleration profile $q_{n}^{(2)}(t)$, to the second order system $G_{\varepsilon}(s)$ characterized by a natural frequency $\omega_{n}$ and a damping factor $\delta$. For this reason in Fig. 15(b) and Fig. 16(b) the spectrum $\left|Q_{n}^{(2)}(j \omega)\right|$ of the acceleration profile, hereafter denoted with $V(\omega)$, is compared with the magnitude of the frequency response of $G_{\varepsilon}(s)$ (properly scaled for the sake of clarity).

From the considerations of Sec.2.3, it follows that the parameters

$$
T_{1}=3 T_{0} \Leftrightarrow \omega_{1}=\frac{\omega_{r}}{3}
$$


and

$$
T_{2}=T_{0} \Leftrightarrow \omega_{2}=\omega_{r}
$$

lead to $\left|M_{1}\left(j \omega_{r}\right)\right|=0$, and $\left|M_{2}\left(j \omega_{r}\right)\right|=0$, and therefore they introduce in $V(\omega)$ a zero of multiplicity two for $\omega=\omega_{r}$. This implies that not only

$$
V\left(\omega_{r}\right)=0
$$

but also

$$
\left.\frac{d V(\omega)}{d \omega}\right|_{\omega=\omega_{r}}=0
$$

and, as a consequence, in the neighborhood of the resonant frequency $\omega_{r}$ the slope of $V(\omega)$ is small and the function remains limited in a broad range of frequencies.

The use of double $\mathrm{S}$ velocity trajectories (with limited jerk) can further improve the result in terms of magnitude of the tracking error as highlighted in a number of works, see (Lambrechts et al., 2005; Barre et al., 2005; Meckl and Arestides, 1998). But also in this case the choice of the filter/trajectory parameters has a strong influence on the system output, as shown in Fig. 17 and Fig. 18 where two double $\mathrm{S}$ velocity trajectories of the same duration are compared. In particular, a choice of the time constants $T_{1}, T_{2}, T_{3}$ that does not take into account the presence of a resonant peak into the system ${ }^{3}$ does not produce any improvement in the tracking performances with re-

\footnotetext{
${ }^{3}$ Note that the values

$$
\begin{aligned}
T_{1} & =\frac{5}{2} T_{0} \Leftrightarrow \omega_{1}=\frac{2}{5} \omega_{r} \\
T_{2}=T_{3} & =\frac{3}{4} T_{0} \Leftrightarrow \omega_{2}=\omega_{3}=\frac{4}{3} \omega_{r}
\end{aligned}
$$
}

do not guarantee that $V\left(\omega_{r}\right)=0$, as shown in Fig. 17(b). 


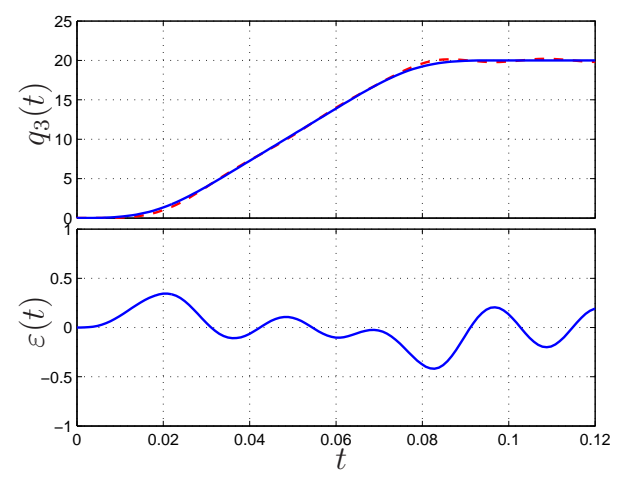

(a)

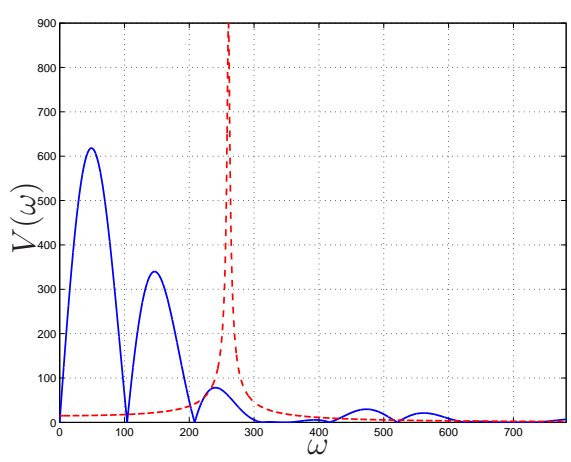

(b)

Figure 17: Response of the elastic system $G_{m l}$ to a double S velocity trajectory obtained with $T_{1}=5 / 2 T_{0}, T_{2}=3 / 4 T_{0}$ and $T_{3}=3 / 4 T_{0}$ : tracking error (a) and frequency spectrum of the acceleration (b).

spect to lower order trajectories such as trapezoidal velocity trajectories, see Fig. 17. On the contrary, by assuming the parameters $T_{1}=2 T_{0}, T_{2}=T_{0}$ and $T_{3}=T_{0}$ the spectrum of the trajectory $V\left(\omega_{r}\right)$ has a zero of multiplicity three for $\omega=\omega_{r}$, and therefore

$$
V\left(\omega_{r}\right)=\left.\frac{d V(\omega)}{d \omega}\right|_{\omega=\omega_{r}}=\left.\frac{d^{2} V(\omega)}{d \omega^{2}}\right|_{\omega=\omega_{r}}=0
$$

From a practical point of view, this means that the values of the function $V(\omega)$ in the neighborhood of the resonant frequency are smaller than those of the trapezoidal velocity trajectory of Fig. 16, whose parameters $T_{i}$ are obtained with similar considerations. As a consequence, the tracking error of the plant is reduced. In particular for the trapezoidal velocity trajectory the maximum value of the error is $\max \left|\varepsilon_{t r}\right|=0.3395$, while for the double $\mathrm{S}$ velocity trajectory $\max \left|\varepsilon_{2 S}\right|=0.2536$, with a reduction $\Delta|\varepsilon| \approx-25 \%$. 


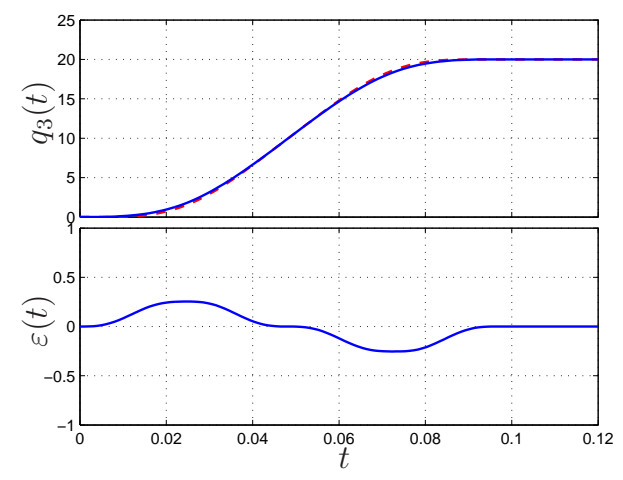

(a)

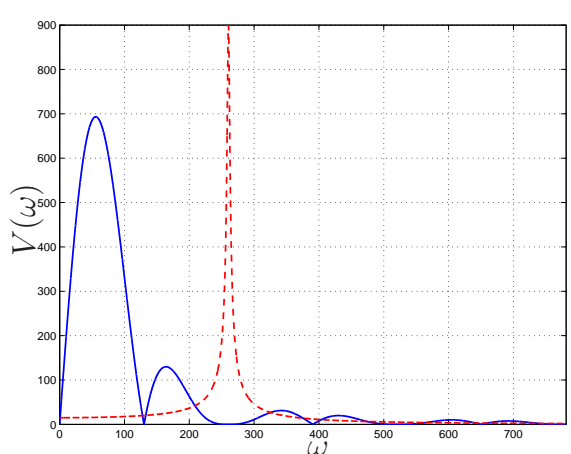

(b)

Figure 18: Response of the elastic system $G_{m l}$ to a double S velocity trajectory obtained with $T_{1}=2 T_{0}, T_{2}=T_{0}$ and $T_{3}=T_{0}$ : tracking error (a) and frequency spectrum of the acceleration (b).

\subsection{Combining time- and frequency-domain specifications}

In the examples discussed so far, only the constraints due to the dynamic behavior of the plant have been considered, while the bounds on velocity, acceleration, etc. have not been taken into account. As a consequence, the peak values of $q^{(1)}(t), q^{(2)}(t)$, etc. depend on the choice of the parameters $T_{i}$. For instance, in the case of the double $\mathrm{S}$ velocity trajectory of Fig. 18(a), the values of such parameters lead to $q_{\max }^{(1)}=|h| / T_{1}=276$ $\mathrm{rad} / \mathrm{s}$ (being the displacement $h=20 \mathrm{rad}), q_{\max }^{(2)}=q_{\max }^{(1)} / T_{2}=11454 \mathrm{rad} / \mathrm{s}^{2}$, $q_{\max }^{(3)}=q_{\max }^{(2)} / T_{3}=474750 \mathrm{rad} / \mathrm{s}^{3}$. However, the interpretation of multisegment trajectories as a bank of filters allows to combine time and frequency constraints. This feature must be profitably exploited in all those cases in which the actuation system imposes some physical limits and the load introduces undesired dynamical modes.

For instance, with reference to the plant $G_{m l}(s)$, if the actuation system 


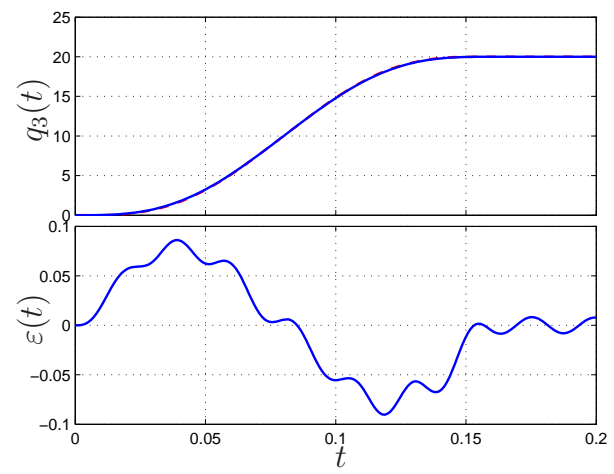

(a)

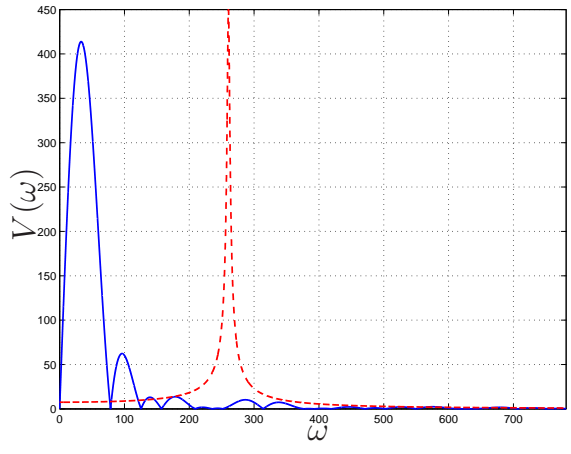

(b)

Figure 19: Response of the elastic system $G_{m l}(s)$ to a double S velocity trajectory obtained with $T_{1}=|h| / q_{\max }^{(1)}, T_{2}=q_{\max }^{(1)} / q_{\max }^{(2)}$ and $T_{3}=T_{1}-T_{2}$ : tracking error (a) and frequency spectrum of the acceleration (b).

is capable of providing a maximum speed $q_{\max }^{(1)}$ and a maximum acceleration $q_{\text {max }}^{(2)}$, a minimum time double $\mathrm{S}$ velocity trajectory can be obtained by assuming $T_{1}=h / q_{\max }^{(1)}$ and $T_{2}=q_{\max }^{(1)} / q_{\max }^{(2)}$, while the parameter $T_{3}$ can be set to the minimum value compliant with constraints (19), that is $T_{3}=T_{1}-T_{2}$. However, although the error is about one order smaller than the error of the trajectories of Fig. 16 and Fig. 18 obtained by tacking into account only the dynamical model of the plant ${ }^{4}$, it exhibits some oscillations when the trajectory stops, as highlighted in Fig. 19(a). In order to reduce these residual vibrations one can set the free parameters $T_{3}$ to $T_{0}$ in order to make $V(\omega)$ null for $\omega=\omega_{r}$, see Fig. 20. In this way, the resonant mode of the plant

\footnotetext{
${ }^{4}$ This is due to the total duration of the trajectory obtained with the time constraints, $T_{t o t}=0.16 \mathrm{~s}$, which is pretty higher than the duration of the other trajectories, $T_{\text {tot }}=$ $0.0965 \mathrm{~s}$. For this reason the magnitude of spectral components at high frequencies is considerably reduced.
} 


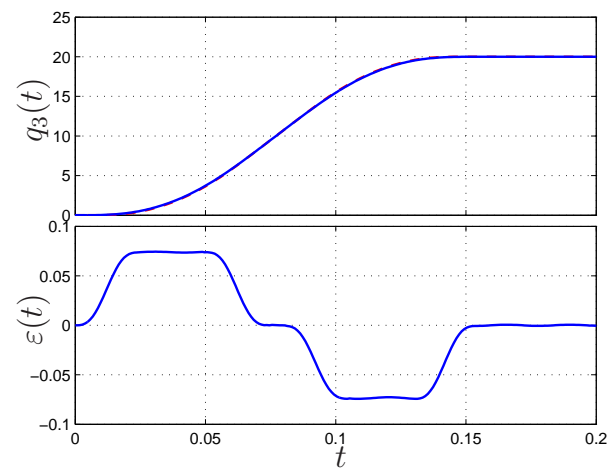

(a)

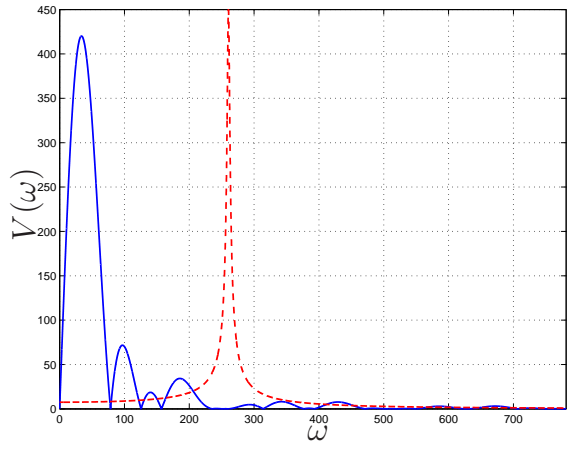

(b)

Figure 20: Response of the elastic system $G_{m l}(s)$ to a double S velocity trajectory obtained with $T_{1}=|h| / q_{\max }^{(1)}, T_{2}=q_{\max }^{(1)} / q_{\max }^{(2)}$ and $T_{3}=T_{0}$ : tracking error (a) and frequency spectrum of the acceleration (b).

is not excited and, at the end of the motion, the error $\varepsilon$ goes immediately to zero. Obviously the bounds on velocity and acceleration are satisfied, as shown in Fig. 21.

If the plant has more than one resonant mode, the parameters of the trajectory should be selected by tacking into account the influence of all dynamical modes, that should be canceled by means of additional filters $M_{i}(z)$.

In conclusion, the formulation of multi-segment trajectories based on dynamic filters allows to consider both time and frequency specifications. In particular, if bounds on the first $m$ derivatives $q_{n}^{(i)}(t), i=1, \ldots, m$ are given, and additionally it is necessary that $V(\omega)=0$ for $l$ critical frequencies $\omega_{r, i}$, $i=1, \ldots, l$, the trajectory order $n$ must be assumed equal to $m+l$. Then, the former $m$ parameters $T_{i}$ must be selected according to (18), while the latter $l$ parameters on the basis of (34). In general, the durations $T_{i}, i=1, \ldots, m$ are higher than $T_{i}, i=m+1, \ldots, m+l$. If this does not occur, it means that 


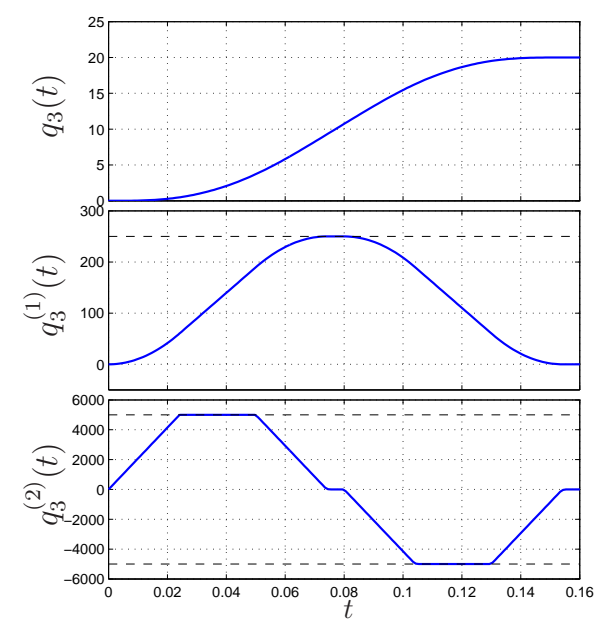

Figure 21: Profiles of position, velocity and acceleration of the trajectory considered in Fig. 20.

the constraints due to dynamical reasons guarantee also the compliance with the limits on velocity, acceleration, jerk, etc. In this case, one can neglect such bounds, that are met in any case because of the frequency constraints, and reduce the order of the trajectory.

\section{Conclusions}

In this paper, the equivalence between multi-segment trajectories and the output of chains of moving average filters has been demonstrated. This result has been the starting point for a generalization of this type of trajectories, that are usually limited to second order (trapezoidal velocity trajectories) or third order (double $\mathrm{S}$ velocity trajectories), to a generic order $n$. In this case the trajectory generator is composed by a cascade of $n$ FIR filters to be fed with a step function signal which defines the desired final position. This 
implies a low complexity and an high efficiency of the trajectory planner, also for large values of $n$. Moreover, its modular structure, composed by linear filters, provides an immediate characterization of the output trajectory from a frequency point of view. This type of analysis allows to define the parameters of the trajectory from frequency considerations and not only on the basis of classical constraints on maximum velocity, acceleration, jerk, etc. Simulative examples help to explain the use of filters for multi-point trajectories planning and the techniques for the design of filters parameters considering both frequency and time specifications.

\section{References}

Barre, P.-J., Béarée, R., Borne, P., Dumetz, E., 2005. Influence of a jerk controlled movement law on the vibratory behaviour of high-dynamics systems. Journal of Intelligent and Robotic Systems 42, 275-293.

Biagiotti, L., Melchiorri, C., 2008. Trajectory Planning for Automatic Machines and Robots. Springer Berlin Heidelberg.

Bonfè, M., Secchi, C., 2010. Online smooth trajectory planning for mobile robots by means of nonlinear filters. In: Intelligent Robots and Systems (IROS), 2010 IEEE/RSJ International Conference on. pp. 4299 -4304.

Fortgang, J., Singhose, W., Mrquez, J. d. J., 2005. Command shaping for micro-mills and cnc controllers. In: Proc. American Control Conference.

Franklin, G. F., Powell, J. D., Workman, M. L., 1998. Digital Control of Dynamic Systems, 3rd Edition. Ellis-Kagle Press. 
Gerelli, O., Guarino Lo Bianco, C., 2008. Real-time path-tracking control of robotic manipulators with bounded torques and torque-derivatives. In: IROS. pp. 532-537.

Gerelli, O., Guarino Lo Bianco, C., 2009. Nonlinear variable structure filter for the online trajectory scaling. Industrial Electronics, IEEE Transactions on 56 (10), $3921-3930$.

Hong, K.-T., Hong, 2004. Input shaping and vsc of container cranes. In: Proc. IEEE International Conference on Control Applications. pp. 1570-1575.

Jeon, J. W., Ha, Y. Y., 2000. A generalized approach for the acceleration and deceleration of industrial robots and cnc machine tools. IEEE Transactions on Industrial Electronics 74, no 1, 133-139.

Kim, D.-I., Jeon, J. W., Kim, S., 1994. Software acceleration/deceleration methods for industrial robots and cnc machine tools. Mechatronics 4 (1), $37-53$.

Lambrechts, P., Boerlage, M., Steinbuch, M., 2005. Trajectory planning and feedforward design for electromechanical motion systems. Control Engineering Practice 13, 145-157.

Magee, D., Book, W., 1998. Optimal filtering to minimize the elastic behavior in serial link manipulators. In: American Control Conference, 1998. Proceedings of the 1998. Vol. 5. Philadelphia, PA, USA, pp. $2637-2642$.

Meckl, P., Arestides, P., 1998. Optimized s-curve motion profiles for minimum residual vibration. In: Proceedings of the American Control Conference. Philadelphia, Pennsylvania, pp. 2627-2631. 
Nozawa, R., Kawamura, H., Sasaki, T., 1985. Acceleration/decelaration circuit.

Olabi, A., Béarée, R., Gibaru, O., Damak, M., 2010. Feedrate planning for machining with industrial six-axis robots. Control Engineering Practice $18(5), 471-482$.

Singer, N., Singhose, W., Seering, W., 1999. Comparison of filtering methods for reducing residual vibration. European Journal of Control 5, 208-218.

Singer, N. C., Seering, W. P., 1990. Preshaping command inputs to reduce system vibration. ASME Journal of Dynamic Systems, Measurement, and Control 112, 76-82.

Singhose, W., 2009. Command shaping for flexible systems: A review of the first 50 years. International Journal Of Precision Engineering And Manufacturing 10 (4), 153-168.

Tuttle, T., Seering, W., 1994. A zero-placement technique for designing shaped inputs to suppress multiple-mode vibration. In: American Control Conference, 1994. Vol. 3. pp. 2533 - 2537.

Zanasi, R., Bianco, C. G. L., Tonielli, A., 2000. Nonlinear filter for the generation of smooth trajectories. Automatica 36, 439-448.

Zanasi, R., Morselli, R., 2003. Discrete minimum time tracking problem for a chain of three integrators with bounded input. Automatica 39 (9), 16431649. 
Zheng, C., Su, Y., Muller, P., 2009. Simple online smooth trajectory generations for industrial systems. Mechatronics 19, 571-576. 